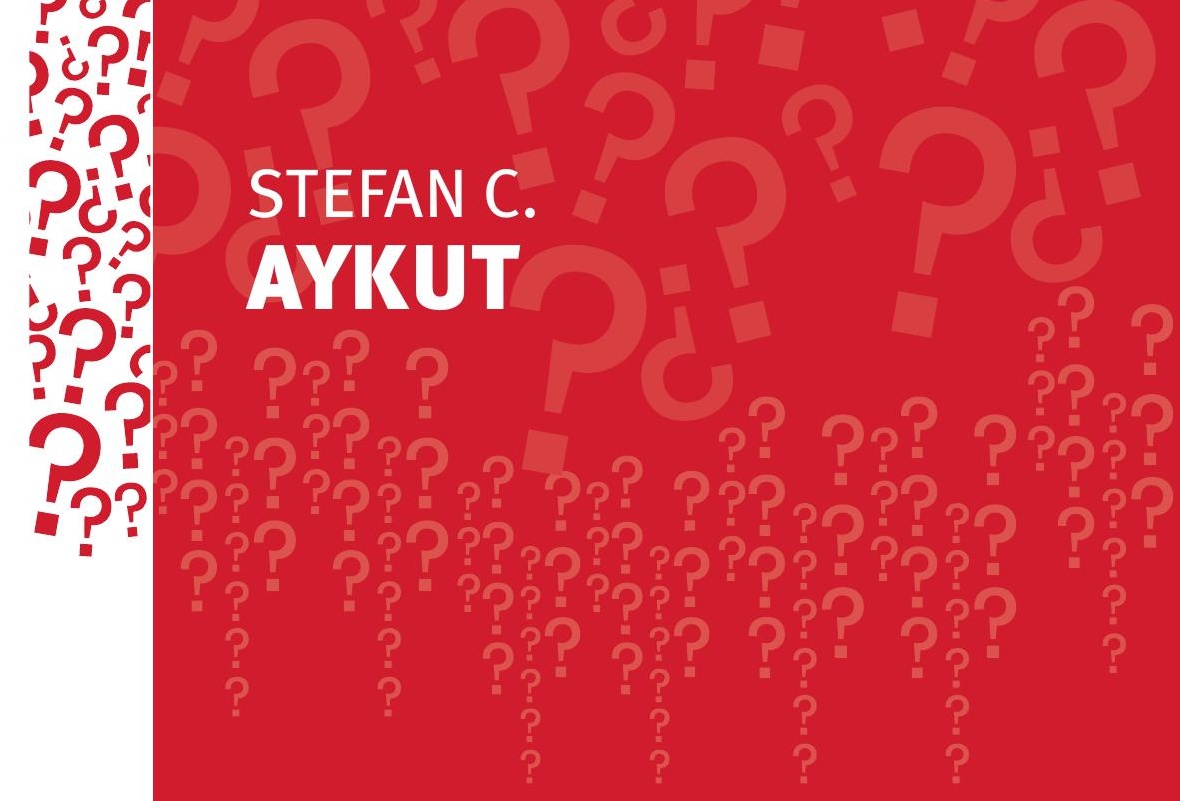

\title{
CLIMATISER LE MONDE
}

๙ั) 



\section{Stefan C. Aykut}

\section{Climatiser \\ le monde}

Conférence-débat organisée par le groupe

Sciences en questions à l'Inra de Paris le 21 juin 2018.

Éditions Quæ, RD 10, 78026 Versailles Cedex 
La collection "Sciences en questions " accueille des textes traitant de questions d'ordre philosophique, épistémologique, anthropologique, sociologique ou éthique, relatives aux sciences et à l'activité scientifique.

\title{
Raphaël Larrère, Catherine Donnars Directeurs de collection
}

Le groupe de travail "Sciences en questions » a été constitué à l'Inra en 1994 à l'initiative des services chargés de la formation et de la communication. Son objectif est de favoriser une réflexion critique sur la recherche par des contributions propres à éclairer, sous une forme accessible et attrayante, les questions philosophiques, sociologiques et épistémologiques relatives à l'activité scientifique.

Texte revu avec la collaboration de Marie-Noëlle Heinrich.

\author{
Éditions Quæ \\ RD 10, 78026 Versailles Cedex \\ (C) Éditions Quæ, 2020
}

ISBN (imprimé) : 978-2-7592-3093-8 ISBN (Pdf) : 978-2-7592-3094-5
ISBN (ePub) : 978-2-7592-3095-2 $1269-8490$

Le code de la propriété intellectuelle du 1er juillet 1992 interdit la photocopie à usage collectif sans autorisation des ayants droit. Le non-respect de cette proposition met en danger l'édition, notamment scientifique. Toute reproduction, partielle ou totale, du présent ouvrage est interdite sans autorisation de l'éditeur ou du Centre français d'exploitation du droit de copie (CFC), 20 rue des Grands-Augustins, 75006 Paris, France. 


\section{Préface}

Le changement climatique renvoie à des sujets souvent abordés dans la collection «Sciences en questions » : le rapport des sciences à la nature, à la technique, les relations entre savoirs et décisions publiques, mais c'est la première fois qu'il est au centre d'un ouvrage. Il était temps! Depuis sa création en 1988, le GIEC (Groupe d'experts intergouvernemental sur l'évolution du climat) précise régulièrement dans ses rapports l'alerte climatique et donne des pistes pour l'atténuer. En 1992, le sommet de la Terre de Rio de Janeiro a instauré un cadre international pour l'action politique sur le climat : la CCNUCC (Convention-cadre des Nations unies sur les changements climatiques) qui réunit maintenant presque tous les pays du monde, qualifiés de "parties», dans des réunions annuelles, les COP (Conferences of the Parties). La première COP s'est tenue à Berlin en 1995. La troisième, à Kyoto en 1997, a obtenu pour la première fois que 37 pays développés s'engagent à réduire leurs émissions de gaz à effet de serre. Après l'échec de la COP de Copenhague en 2009, celle de Paris en 2015 a semblé relancer la dynamique internationale, de par le nombre de pays impliqués (195), de par l'ampleur des sujets abordés, de par l'ambition politique affichée de limiter la hausse «bien en deçà de $2^{\circ} \mathrm{C} »$, en avançant le chiffre de $1,5^{\circ} \mathrm{C}$, qui exclut toute poursuite de la course actuelle du monde. Mais l'accord de Paris qui a suivi en 2016 a également été marqué par le retrait des États-Unis, éclairant d'un jour nettement moins engageant l'échiquier politique du climat.

Malgré la récurrence des dénégations du changement climatique et le retrait américain, le climat s'est immiscé dans nos vies et nos réflexions : il touche nos modes de vie et de consommation, nos sensibilités face aux orientations politiques, nos pratiques professionnelles... De cette «climatisation du 
monde», Stefan C. Aykut, professeur junior à l'université de Hambourg est un observateur et un analyste attentif.

Stefan, tu as passé ton enfance à Stuttgart où tu as étudié le français au lycée, obtenant un double bac franco-allemand, l'Abibac. Ce choix revient à la proximité entre le lycée qui le proposait et ta maison et non à des attaches particulières avec la France; tes attaches familiales mêlent la Turquie et l'Allemagne. Le bac en poche, tu pars étudier les sciences politiques à Berlin. Et grâce à Erasmus, tu viens en France en 2004 étudier à l'EHESS. Tu découvres la variété des enseignements en sciences sociales. Pouvoir ainsi croiser les disciplines, passer des sciences politiques à la sociologie, de l'histoire à l'anthropologie te plait particulièrement. Tu t'orientes alors vers un domaine de recherche multidisciplinaire des sciences sociales, les Science and Technology Studies, les STS, lesquelles éclairent par de multiples voies et objets, les relations entre les sciences, les technologies et la société. Tu suis d'abord un master en STS, au Centre Alexandre Koyré (EHESS), puis y poursuis une thèse sur la construction du changement climatique comme problème public. Ta thèse comporte une comparaison des politiques énergétiques entre la France et l'Allemagne, et une analyse de la mise en place de la gouvernance globale du climat. Ce travail te montre, entre autres, combien les décisions et les orientations prises dépendent de choix passés, d'où l'importance de prendre en compte la durée dans notre façon d'aborder toute négociation internationale. Ta directrice de thèse est Amy Dahan, historienne des sciences, directrice de recherche émérite au CNRS rattachée au Centre Alexandre Koyré. Avec elle, tu as fait un bout de chemin pionnier et original, pour faire du changement climatique une question sociale, une matière à penser dans les sciences sociales. De cette collaboration fructueuse, vous tirerez un livre - Gouverner le climat? 20 ans de négociations internationales - qui est devenu une référence sur le sujet. Il introduit la notion de régime climatique, en tant que clé de lecture du changement global et de la façon d'y faire face. 
Depuis une dizaine d'années donc, jonglant entre un postdoctorat à l'Ifris à Marne-la-Vallée, des contrats avec le centre Marc Bloch à Berlin et des programmes ANR, tu es devenu un fin observateur des négociations internationales sur le changement climatique. Tu as notamment scruté le déroulement, les jeux d'acteurs, les discours et les actes au cours de la COP 21 à Paris. Avec Jean Foyer (CNRS) et Édouard Morena, vous avez synthétisé les observations d'un groupe de chercheurs dans un livre, Globalising the climate: COP 21 and the climatisation of global debates. Vous y développez comment la gouvernance du climat a inclus dans son arène progressivement de nouveaux enjeux et de nouveaux acteurs. C'est ce phénomène que vous avez appelé la «globalisation du climat». Celui-ci se double d'un autre phénomène, la «climatisation du monde», c'est-à-dire l'emprise des enjeux climatiques sur les autres enjeux, lesquels sont dorénavant regardés à travers la focale du climat. Les deux phénomènes interagissent.

Curieux des croisements entre approches, tu t'es intéressé récemment au rôle des acteurs financiers dans les politiques de lutte contre le changement climatique, ce que disent - et cachent sans doute aussi - leurs propositions. Et aussi comment les questions climatiques sont appréhendées par le droit. Tu as été l'un des organisateurs d'un colloque international qui s'est tenu fin mai 2018, à l'ENS à Paris, sur «Les bons usages de la terre».

Ton regard sur les évolutions en cours nous semble stimulant pour penser comment le changement climatique interpelle les sciences dans leur responsabilité et éthique. Mais commençons par le début, dis-nous comment s'est opérée la «climatisation» du monde?

Catherine Donnars Co-directrice de la collection «Sciences en questions» 



\section{Climatiser le monde}

\section{Introduction}

Le point de départ de mon propos ${ }^{1}$ est une observation simple : au cours de la dernière décennie, la question du changement climatique a changé de statut. Quand je commençais à m'y intéresser dans le cadre de mes recherches, au milieu des années 2000, il s'agissait d'un sujet un peu exotique qui animait à peine plus qu'un petit cercle de chercheurs, militants, diplomates et professionnels de l'environnement. Depuis, le climat est progressivement devenu un enjeu politique de premier plan et une thématique transversale, qui irrigue les débats sociétaux, stimule des activités de recherche et provoque des repositionnements d'acteurs dans des domaines très variés. Prenons l'exemple des villes. Celles-ci ont non seulement créé des réseaux transnationaux comme le Cities for Climate Protection program (1993) et le C40 Cities Climate Leadership Group (2005) pour coordonner leurs efforts en termes de réduction des émissions et d'adaptation au réchauffement. À coup de sommets médiatiques, prises de parole publiques et publications régulières, les gouvernements municipaux se posent aussi en hérauts de la protection du climat et revendiquent un rôle plus important dans la gouvernance globale. Sur un mode opératoire similaire, des milieux industriels «verts» ont récemment fondé We Mean Business, une alliance de firmes et de think tanks qui se veulent progressistes sur le climat. Dans son premier rapport Le climat a changé (We Mean Business, 2014) et une longue série de publications

1. Les thèses défendues dans ce livre sont le fruit de recherches collectives, menées au sein de l'équipe «Changement climatique et biosphère» dirigée par Amy Dahan au Centre Alexandre Koyré (EHESS), et dans le cadre du projet ClimaCop (Ifris) dirigé par Jean Foyer, Edouard Morena et moi-même. 
ultérieures, l'alliance plaide pour une prise en compte systématique de l'empreinte carbone dans l'activité économique, mais aussi pour des régulations plus strictes. Ce positionnement rompt clairement avec la préférence traditionnelle des lobbies économiques pour des approches incitatives et non contraignantes. Du côté du monde religieux, Loué sois-tu, la première encyclique entièrement dédiée aux questions écologiques, défraye la chronique en 2015. Le pape François $\mathrm{y}$ dresse un constat alarmant de la dégradation de la planète par les activités humaines. Le réchauffement climatique et ses effets occupent une place centrale dans ce récit. Des évolutions similaires ont lieu dans d'autres religions, même si celles-ci ne rencontrent pas toujours un écho comparable dans les médias occidentaux. Ainsi, on compte désormais des déclarations musulmane, bouddhiste, hindou, sikh, juive, protestante, etc., sur le climat ${ }^{2}$. Les milieux de la finance ne sont pas épargnés. À coup de slogans tels que Divest Wall Street, Divest Harvard ou Paris Zéro Fossile, des groupes d'activistes demandent aux organismes publics et investisseurs privés de se séparer de leurs actifs dans le secteur des énergies fossiles et d'adopter des stratégies d'investissement bas-carbone. En septembre 2015, ils trouvent un allié de circonstance plutôt inattendu dans le gouverneur de la banque d'Angleterre, Mark Carney. Dans un discours très remarqué, titré «Briser la tragédie de l'horizon», celui-ci alerte alors sur les risques que le changement climatique ferait peser sur le système financier mondial, et enjoint aux banques et aux investisseurs institutionnels de mieux prendre en compte les implications à long terme d'une transition mondiale bascarbone. Ces évolutions dans différents domaines culminent en décembre de la même année à la conférence climatique COP21 à Paris. Durant les deux semaines de l'évènement,

2. Ce site recense les principales déclarations : $<$ https://www.interfaithpowerandlight.org/religious-statements-on-climate-change/> (consulté le 02 juillet 2019). 
les entreprises exhibent leurs innovations technologiques et visions du futur à l'exposition «Solutions COP21» au Grand Palais et à «la Galérie», un espace dédié proche du centre de conférences onusien au Bourget. En parallèle, des représentants de peuples autochtones défilent dans Paris pour promouvoir leurs savoirs traditionnels dans la lutte contre les effets du réchauffement, et une coalition hétéroclite de mouvements sociaux et d'associations se retrouve au " Village mondial des alternatives» à Montreuil autour d'innovations sociales et de solutions low tech. Depuis Paris, le phénomène semble s'être encore intensifié. De moins en moins de champs d'activité échappent désormais à l'emprise de l'enjeu climatique. À la recherche de leviers pour réduire les émissions, d'aucuns proposent de nouvelles stratégies de développement urbain (climate smart cities), promeuvent des techniques agricoles bas-carbone (climate smart agriculture), ou militent pour aligner les flux financiers publics et privés avec l'impératif de réduction (climate finance). D'autres alertent que les impacts prévisibles du réchauffement nous forcent à repenser en profondeur les projets de construction et stratégies d'aménagement du territoire (climate proofing), les politiques migratoires (climate migrations) ou encore les enjeux de sécurité internationale (climate security).

Pris ensemble, ces exemples dressent le tableau général d'une diffusion de la question climatique dans des sphères de plus en plus nombreuses et variées de la vie publique. Les effets combinés - actuels ou prospectifs — du réchauffement en cours et des mesures de riposte forcent des acteurs souvent assez éloignés des enjeux écologiques à s'intéresser à la question et à évaluer son impact sur leurs activités. En nombre croissant, firmes, associations, administrations et organismes de recherche se voient ainsi contraints de repenser leurs orientations stratégiques, leurs routines organisationnelles et leurs pratiques économiques à l'aune de la question climatique. Cette extension du domaine de la gouvernance du climat 
constitue un phénomène assez inédit, qui amène une série de questionnements. Comment expliquer cette dynamique : où trouve-t-elle sa source et qu'est-ce qui constitue sa force motrice? Quels sont ses caractéristiques et modes de déploiement? Doit-on y voir — enfin! — le signe d'une meilleure prise en charge du problème climatique, voire d'une transition écologique en cours? Un regard sur l'évolution des émissions mondiales, qui ont atteint un nouveau record en 2018, permet d'en douter ${ }^{3}$. Comment alors faire sens du phénomène et évaluer ses effets, d'une part sur la gouvernance climatique, et d'autre part sur les autres domaines et activités concernés? Ces questions forment le fil rouge de mon propos.

Dans ce qui suit, j'introduis d'abord mon hypothèse centrale, qui propose de saisir les évolutions en cours comme résultant du double mouvement d'une "globalisation du climat» et d'une «climatisation du monde». Les trois sections suivantes s'intéressent aux origines et aux causes de ce double mouvement. Je débute par un regard historique sur la gouvernance globale du climat, qui montre que ses impasses et ses échecs constituent une des sources profondes du phénomène. Je poursuis par une étude sur la COP21, pour illustrer la force d'attraction des sommets onusiens comme un vecteur-clef de la dynamique de climatisation. Je conclus cette partie par une réflexion sur le nouveau mode de gouvernance mis en place à Paris. Comme celui-ci repose sur une mobilisation tous azimuts de la société civile mondiale, il se nourrit du phénomène de climatisation et contribue à son accélération récente. Les sections subséquentes braquent le projecteur sur deux domaines spécifiques, fortement affectés par la montée en puissance de la question climatique : les politiques

3. Des chiffres actuels sur l'évolution des émissions et leur trajectoire provisionnelle (en fonction des politiques climatiques nationales) sont disponibles sur le site Climate Action Tracker : <https://climateactiontracker.org> (consulté le 02 juillet 2019). 
énergétiques et les recherches en sciences du sol. Ces études de cas visent à mieux cerner les caractéristiques du processus de climatisation, ses modes de déploiement et ses effets. Elles illustrent qu'il s'agit d'un processus à multiples facettes, qui prend différentes formes et intensités, et peut avoir des effets ambigus et contradictoires. Elles montrent aussi que rien ne garantit qu'en fin de compte, la dynamique de climatisation s'accompagne effectivement d'une meilleure gestion du problème climatique.

\section{La thèse de la climatisation : du constat empirique au prisme analytique}

La thèse au cœur de cet ouvrage est d'abord le fruit d'une rencontre entre deux équipes de recherche. La première, dont je faisais partie, s'est constituée autour de l'historienne des sciences Amy Dahan au Centre Alexandre Koyré de l'EHESS. Nous avions travaillé sur la gouvernance globale du climat, son histoire institutionnelle et politique, et les relations science-politique qui la caractérisent ${ }^{4}$. Au cours de ces recherches, nous avions été frappés par le fait que les négociations, et encore plus les débats en marge du processus politique, brassaient des sujets de plus en plus divers, dont certains pouvaient paraître assez éloignés du cœur du problème : l'énergie et le développement bien sûr, mais aussi l'équité Nord-Sud, l'aménagement urbain et la construction, l'agriculture et l'usage des sols, la gestion des forêts et l'usage des terres... Souvent, ces thématiques apparaissaient d'abord dans des rapports et des évènements parallèles - ou side-events - organisées au moment des grands sommets onusiens, avant de parfois faire leur entrée dans le cœur des négociations. Menée par Jean Foyer de l'Institut des sciences

4. Voir nos comptes rendus réguliers de missions d'observation de sommets onusiens (Dahan et al., 2009; Dahan et al., 2010; Dahan et Aykut, 2012), ainsi que notre ouvrage sur l'histoire des négociations (Aykut et Dahan, 2015). 
de la communication du CNRS (ISCC), l'autre équipe s'était constituée de manière informelle lors d'une mission d'observation à la conférence onusienne Rio +20 sur le développement durable, tenue à Rio de Janeiro au Brésil en 2012 (Foyer, 2015). Elle réunissait des chercheuses et chercheurs d'institutions diverses, qui s'étaient plutôt intéressés à d'autres arènes et d'autres sujets internationaux, comme la gouvernance de la biodiversité et de l'agriculture, ou encore à la constitution de nouveaux acteurs globaux, comme les peuples autochtones ou le business. Leur intérêt pour le climat était donc plus récent. Il se nourrissait d'un constat qui faisait écho au nôtre, tout en partant d'une perspective différente : leurs domaines d'étude étaient en train de se transformer sous leurs yeux. De plus en plus d'acteurs de ces domaines commençaient à s'intéresser à la question climatique, que ce soit parce que les effets du réchauffement affectaient leurs espaces de vie et leurs activités économiques, ou pour se positionner dans la course aux solutions bas-carbone, et ainsi profiter de l'espace politique et économique ouvert par les transformations à venir.

La conjonction de ces deux constats nous intriguait en amont de la COP21, alors que nous étions en train de monter un projet collaboratif pour observer la conférences. Nous avons donc structuré notre recherche autour d'une double hypothèse (Foyer et al., 2017) : d'une part, nous assistons à une globalisation du climat, qui s'opère par l'inclusion de nouveaux enjeux et l'association de nouveaux acteurs à la gouvernance climatique. Celle-ci rend la formulation de politiques climatiques de plus en plus complexe, que ce soit dans les administrations locales et nationales, ou dans les négociations onusiennes. D'autre part, nous observons une climatisation du monde, qui se traduit par l'extension du problème climatique vers d'autres arènes politiques. En conséquence, des acteurs aux

5. Pour plus d'informations sur le projet ClimaCop, voir : $<$ https://climacop.hypotheses.org > (consulté le 02 juillet 2019). 
agendas très divers sont amenés à «climatiser» leurs discours et les enjeux qu'ils portent, afin de les mettre en relation avec la thématique du climat. Ces deux dynamiques ne sont pas indépendantes; elles se répondent, se conjuguent, peuvent se renforcer mutuellement. Ensemble, elles traduisent l'idée selon laquelle la question climatique présente désormais un certain pouvoir agrégatif pour toute une série de thématiques connexes. La perspective analytique adoptée est néanmoins importante. Selon qu'elle cible d'abord l'arène climatique et ses acteurs, ou au contraire d'autres domaines affectés par la question climatique, elle rend visible d'autres processus sociaux, et braque le projecteur sur d'autres acteurs et enjeux.

À la recherche des causes profondes et des principaux vecteurs de ce double mouvement, une première piste pointe vers la nature, intrinsèquement transversale et interconnectée, du problème climatique. Cela concerne d'abord les caractéristiques du climat comme objet scientifique. En climatologie, «everything is connected to everything else» notent les chercheurs Stephen Schneider et Lynne Mesirow (1976, 117), pour résumer leur expérience de scientifiques : quand on veut étudier le système climatique, il faut prendre en compte une série potentiellement infinie d'autres phénomènes et processus, des cycles de l'eau et du carbone en passant par les interactions océan-atmosphère et les oscillations des courants océaniques, jusqu'aux dynamiques des écosystèmes et leurs réactions à des modifications environnementales. Très tôt, la climatologie a donc dû puiser dans les résultats de recherches menées dans d'autres disciplines. La relation qu'elle entretenait avec ces disciplines s'est d'ailleurs progressivement inversée, dans la mesure où l'accroissement des financements publics consacrés à la prévision du réchauffement en cours conférait un statut de plus en plus central à la modélisation climatique. Des chercheurs d'autres domaines - et parfois des sous-disciplines entières — ont alors été attirés dans le giron des recherches sur le climat. La transversalité de la 
question climatique provient, ensuite, de la multifactorialité de ses causes et donc de la complexité qui caractérise toute tentative de formuler une riposte politique au problème. This changes everything - ce titre d'un ouvrage de Naomi Klein (2014) - résume bien l'enjeu. Si on veut réellement combattre le changement climatique, il faut changer tout : nos routines quotidiennes, nos habitudes de consommation et modes de déplacement bien sûr, mais aussi nos systèmes énergétiques, les processus industriels et les modes de gestion des firmes, voire notre modèle économique et les règles du commerce mondial. Un diagnostic similaire est formulé par le sociologue Bruno Latour (2015). Selon lui, nous sommes entrés dans un «nouveau régime climatique ${ }^{6} »$, qui nous force à repenser les institutions fondatrices - politiques et économiques mais aussi philosophiques et spirituelles — de la modernité industrielle. Le caractère totalisant et englobant de la question climatique forme donc l'arrière-plan devant lequel se déploient les dynamiques de globalisation du climat et de climatisation du monde. Celles-ci représentent deux réponses possibles, mais diamétralement opposées, à l'imbroglio de la lutte contre le changement climatique : soit les négociations climatiques intègrent de plus en plus d'acteurs et d'enjeux divers, au risque de devenir «obèses» et potentiellement ingérables; soit l'enjeu climatique est diffusé dans un nombre croissant d'autres arènes de gouvernance et intégré aux routines de gestion d'autres problèmes, au péril toutefois de rester marginal dans ces arènes, voire d'être dilué dans ce processus?

6. Le terme de «régime climatique» revêt ici un sens beaucoup plus large que dans l'usage qui en est fait en relations internationales (voir ci-dessous).

7. Cette tension transparaît aussi dans la littérature en relations internationales, où les tentatives d'établir des liens entre la gouvernance climatique et d'autres arènes globales ont été discutées de manière positive sous le terme de «climate bandwagoning» (Jinnah, 2011), tandis que le «verdissement» du discours des organisations internationales a été critiqué comme une forme de "climate mainstreaming», dont les effets concrets resteraient souvent très limités (Methmann, 2010). 
Une des sources ou raisons profondes de l'extension du domaine de la gouvernance climatique réside donc dans la nature même du problème. Or celle-ci n'explique pas comment ce processus s'opère concrètement, quels acteurs le portent et quels domaines il concerne. Une telle vision n'aide pas non plus à identifier les raisons de l'accélération et de l'intensification récente du phénomène. Pour le comprendre, il faut déplacer le regard, de la nature du problème aux dynamiques sociales qui caractérisent et accompagnent son traitement politique. Cela nous amène au sujet de la gouvernance globale du climat.

La Convention cadre des Nations unies sur le changement climatique de 1992 - ou Convention climat - forme le cadre institutionnel de cette gouvernance. Il s'agit d'un traité universel, puisque la quasi-totalité des pays du monde en sont membres. Les négociations sur le climat réunissent donc chaque année des représentants de pays du monde entier. Au fil des années, ces sommets mondiaux - conférences des parties, ou COPs dans le jargon onusien - sont venus constituer un rendez-vous politique de tout premier plan, qui jouit d'une attention médiatique considérable. Ainsi, 117 chefs d'État et de gouvernement ont assisté à la COP21 à Paris en 2015, une première dans l'histoire des sommets onusiens. À la lumière de cette évolution, on comprend mieux pourquoi ces conférences exercent, bien au-delà du seul monde politique et des milieux écologiques, une force d'attraction importante sur tout un ensemble d'acteurs d'autres domaines. Parmi ceux-ci, certains voient l'arène climatique comme un point de passage obligé pour faire entendre leur voix et promouvoir leurs intérêts au niveau global. D'autres - parfois les mêmes - veulent surtout profiter des sources de financement offertes dans ce cadre, ou espèrent accéder à une forme de reconnaissance symbolique en participant aux instances de la gouvernance globale. En somme, il faut donc s'intéresser 
de plus près aux enjeux et à l'évolution de la gouvernance globale du climat pour mieux comprendre ce qui constitue la force motrice du processus de climatisation et d'où il tire sa dynamique récente.

\section{Les sources historiques du processus de climatisation}

Dans la littérature, le terme «régime» désigne les arrangements politiques, traités, normes et organisations internationales créés pour réguler un domaine spécifique des relations internationales (Krasner, 1983). Le «régime climatique» regroupe donc les institutions de gouvernance mises en place pour lutter contre le réchauffement global et combattre ses effets. Dans une acception élargie, il inclut également les relations science-politique et arrangements marchands qui caractérisent le domaine (Aykut et Dahan, 2015). Une dimension importante de la notion de régime est qu'elle évoque l'idée d'une continuité dans le temps des institutions de gouvernance. C'est une perspective de longue durée, qui souligne l'importance des phénomènes de dépendance au sentier, que ce soit à travers la permanence des cadrages cognitifs, la persistance des routines organisationnelles, ou la pérennité des normes juridiques. Bref, les régimes internationaux se construisent progressivement et par couches successives, et il n'est pas aisé de changer un tel régime, une fois qu'il est établi.

\section{Une brève histoire de la gouvernance globale du climat}

Le régime climatique se met en place au tournant des années 1990. La première organisation créée dans le cadre des négociations internationales est un organisme d'expertise : le Groupe d'experts intergouvernemental sur l'évolution du climat (GIEC), mis en place en 1988 par l'Organisation météorologique mondiale et le Programme des Nations unies pour l'environnement. Il a pour mission d'être «policy relevant but not policy prescriptive», c'est-à-dire de fournir 
une base scientifique aux négociations internationales, tout en restant neutre quant au choix des mesures à prendre ${ }^{8}$. D'apparence simple, au moins en théorie, cette maxime s'est avérée beaucoup plus compliquée à mettre en œuvre dans la pratique. Comment caractériser un problème et ses causes, sans aussi suggérer, au moins implicitement, les voies qui permettraient de le résoudre? La production la plus importante du GIEC sont les rapports de synthèse publiés peu ou prou tous les cinq ans. Ces rapports sont préparés par trois groupes différents, qui compilent respectivement l'état des connaissances sur le système climatique et son évolution (groupe 1), sur les impacts du réchauffement et les mesures d'adaptation (groupe 2), et sur les options pour la réduction des émissions (groupe 3). De manière générale, la modélisation numérique occupe une place très importante dans ces recherches. Ainsi, des modèles de circulation générale (General Circulation Models) sont utilisés pour simuler l'évolution du climat global, tandis que des modèles économiques et modèles d'évaluation intégrée (Integrated Assessment Models) fournissent la base des estimations sur l'évolution des émissions, et fondent l'évaluation de différentes mesures de riposte. Grâce à leur position centrale dans l'expertise, ces modèles structurent la collaboration entre disciplines. Leur prédominance renforce aussi un biais existant en faveur des savoirs quantifiés dans les débats climatiques, ce qui n'est pas sans importance pour les domaines académiques attirés dans le giron des recherches sur le climat.

$\mathrm{Au}$ niveau politique, la conférence des Nations unies sur le développement durable à Rio de Janeiro en 1992 constitue une première étape décisive. Elle débouche sur la signature de la Convention climat, qui établit un processus de

8. Mike Hulme et Martin Mahony (2010) présentent un état des lieux des recherches en sciences sociales sur le GIEC. Amy Dahan et Hélène Guillemot (2015) discutent des évolutions plus récentes. 
négociations avec des rencontres annuelles - les COPs. Elle définit aussi les principes fondateurs du régime climatique. Ainsi, l'article 2 du traité stipule que «l'objectif ultime» des négociations est de stabiliser les concentrations de gaz à effet de serre «à un niveau qui empêche toute perturbation anthropique dangereuse du système climatique $»$. Mais où se situe ce seuil «dangereux»? Tout sauf anodine, cette question constituera un enjeu majeur des débats scientifiques et politiques pour les décennies à venir. Dans un processus complexe qui mêle expertise et politique, ces débats convergent à la fin des années 2000 sur l'objectif des $2^{\circ} \mathrm{C}$ - contenir l'augmentation de la température moyenne du globe à $2^{\circ} \mathrm{C}$ par rapport à l'ère préindustrielle (Aykut et Dahan, 2011). L'article 4 de la convention fixe un deuxième principe important : celui des «responsabilités communes mais différenciées», selon lequel les pays industrialisés doivent montrer le chemin en réduisant leurs émissions en premier, tout en fournissant de l'aide aux pays en voie de développement afin que ceux-ci puissent s'adapter et adopter des modes de développement plus sobres en carbone. Sur la base de ce principe, une séparation stricte est alors établie entre pays du Nord et pays $\mathrm{du}$ Sud, et les négociations concernent d'abord principalement les premiers. Le protocole de Kyoto, signé à la COP3 au Japon en 1997, entérine un peu plus cette séparation. Il assigne des objectifs chiffrés de réduction des émissions aux pays industrialisés, alors que les autres pays en sont exemptés.

Dès ses débuts, et malgré les succès diplomatiques à Rio et à Kyoto, la gouvernance climatique est aussi traversée par des clivages profonds et caractérisée par des défaillances importantes. Ainsi, le président américain George Bush vient à la conférence de Rio en déclarant que «the American way of life is not negotiable» - le mode de vie américain n'est pas négociable. On peut donc parler de tout, mais il n'est pas question de s'attaquer au cœur du problème : les modes de vie énergivores et insoutenables des pays du Nord, et tout particulièrement 
des États-Unis, alors premier émetteur mondial de gaz carbonique. Son fils George W. Bush réaffirme ce positionnement dix ans plus tard, durant la campagne présidentielle américaine de 2000. Alors que le monde attend que les États-Unis ratifient enfin le protocole de Kyoto, le candidat républicain clame son opposition à un accord qui, selon lui, forcerait les Américains à «marcher [pour aller] au travail» (Bailey, 2016, 90). Loin d'être isolée, la position du futur président reflète alors celle d'une partie significative de la classe politique étatsunienne, comme l'illustre la fameuse résolution Byrd-Hagel de 1997 votée à l'unanimité (!) par le Sénat américain. Dans cette résolution, les sénateurs avertissent qu'ils ne ratifieront pas un traité qui fixerait des réductions chiffrées aux ÉtatsUnis sans demander des efforts «comparables» aux pays en développement. Bien entendu, cette condition était totalement inacceptable pour les pays du Sud, et ne reflétait pas l'esprit des négociations en cours. Après sa prise de fonction en 2001, George W. Bush décide par conséquent de se retirer du protocole de Kyoto. Cette première défection des États-Unis ne restera pas la seule : l'histoire se répète quinze ans plus tard, quand Donald Trump, fraîchement élu, annonce sa volonté de se retirer de l'accord de Paris.

\section{Un schisme au cœur de la gouvernance onusienne}

La période qui s'ouvre après le retrait des États-Unis du protocole de Kyoto débute néanmoins par une lueur d'espoir. L'Union européenne réussit à convaincre suffisamment de pays de ratifier le protocole pour assurer son entrée en vigueur et ainsi le sauver. Cette victoire est toutefois obtenue au prix de concessions, qui affaiblissent durablement l'efficacité environnementale du protocole. Autre conséquence du retrait américain, les négociations se complexifient : d'une part, elles se scindent en deux, puisque la nouvelle voie de négociations ouverte sous le protocole de Kyoto vient s'ajouter à celle, existante, sous la Convention climat. Cette 
dernière est préservée afin de ne pas fermer la porte aux Américains et permettre leur retour à un moment politique plus opportun. D'autre part, le vide laissé par les États-Unis permet au G77+Chine, alliance historique des pays du Sud, d'assumer un rôle plus important dans les négociations. Ils y promeuvent tout un ensemble de nouveaux sujets en ligne avec leurs intérêts, comme l'adaptation et le transfert financier, mais aussi des questions d'agriculture et d'usage des terres, de biodiversité et de gestion des forêts, ou encore le transfert de technologies. C'est une des origines du phénomène de globalisation du climat. La période se clôt sur l'échec retentissant de la conférence de Copenhague en 2009. À cette conférence, on devait s'accorder sur un nouveau traité pour remplacer le protocole de Kyoto. La déception est d'autant plus profonde que l'élection de Barack Obama à la présidence des États-Unis peu avant la conférence avait nourri des espoirs de changement de cap dans la diplomatie américaine. Or il n'en fut rien, ou presque. Au lieu du grand soir du climat escompté, Copenhague devient le théâtre de l'avènement d'un nouvel ordre géopolitique mondial. Au dernier jour de la conférence, l'Europe est complètement mise de côté, alors que les États-Unis négocient directement avec la Chine et d'autres puissances émergentes (Grubb, 2010)9 . Le soi-disant «accord de Copenhague» qui en résulte - en fait, un bout de papier de trois petites pages non officiellement adopté par la $\mathrm{COP}$ - dessine une approche entièrement basée sur le volontariat, qui rompt définitivement avec le modèle contraignant du protocole de Kyoto.

L'échec de la voie de Kyoto à Copenhague n'était pas contingent. Durant la première décennie du nouveau millénaire, le régime climatique était traversé par des contradictions de plus en plus flagrantes, une situation que nous avons qualifiée de «schisme de réalité» (Aykut et Dahan,

9. Le Brésil, l'Inde et l'Afrique du Sud. 
2014). Ce terme décrit d'abord le ressenti d'un décalage croissant : d'une part, des conférences climatiques régies par la civilité onusienne, la recherche de consensus et une reconnaissance quasi-universelle de l'urgence climatique; d'autre part, un système international marqué par la persistance de modes de développement très carbonés, la concurrence effrénée pour des parts de marché et une recrudescence de la violence militaire. À la recherche de facteurs qui permettraient d'affiner cette première intuition et de mieux comprendre ses causes, nous avons distingué plusieurs dimensions du schisme (Aykut, 2016). Premièrement, le problème climatique a été pensé, par les climatologues et les économistes, comme un problème d'emblée et irréductiblement global. Face à la catastrophe annoncée, nous serions toutes et tous également concernés, tous dans le même bateau. Et pour résoudre le problème, il faudrait un effort de coopération collectif, qui inclut tous les pays du monde. Ce cadrage comme «problème d'action collective» correspond à une forme spécifique de prise en charge politique. Ainsi, le cadre onusien a longtemps été considéré comme la seule arène légitime, et un traité international contraignant comme seul moyen efficace pour combattre le changement climatique. Cela marginalisait en revanche les efforts d'acteurs non-étatiques, les réseaux de villes et initiatives privées, ou encore les «clubs» de pays plus restreints qui se réunissent sur des sujets spécifiques. Deuxièmement, la question climatique a été abordée comme un problème de pollution. Les images d'ours polaires ou de cheminées laissant échapper d'épais nuages de fumée, utilisées dans les campagnes d'ONGs et les médias, sont édifiantes à cet égard. Ces images rapprochent le climat d'autres problèmes environnementaux causés par les émissions industrielles, comme les «pluies acides» et les pollutions transfrontalières, ou le «trou» dans la couche d'ozone. Au moment de la mise en place des institutions de la gouvernance climat, on va donc caler ceux-ci sur l'approche 
choisie pour la régulation de ces autres problèmes. Ce modèle repose sur des mesures dites «en fin de tuyau», c'est-à-dire qu'on va tenter de réguler les outputs, et donc les émissions de gaz à effet de serre. Les négociations internationales concerneront donc principalement la répartition de l'effort global de réduction des émissions. On ne s'attaquera pas, en revanche, à la question des inputs, c'est-à-dire aux processus qui déterminent l'évolution des émissions, comme la production énergétique, les modèles de développement industriel ou le fonctionnement de l'économie mondiale. Cette approche a aussi favorisé, troisièmement, l'enclavement de la gouvernance climatique sur l'échiquier international, en la séparant de tout un ensemble d'autres arènes et régimes internationaux. Alors que le fonctionnement des marchés énergétiques mondiaux a été identifié comme une des «causes profondes» du problème, la régulation de ces marchés ne fait pas partie des sujets débattus dans les négociations climatiques (Victor, 2011, 19). D'autres enjeux cruciaux sont absents de ces pourparlers : la régulation du commerce mondial est traitée au sein de l'Organisation mondiale du commerce ou dans des négociations bilatérales; et la régulation des marchés financiers se négocie au sein d'arènes confidentielles comme le Comité de Bâle sur le contrôle bancaire. Ces arènes ne prennent pas du tout en compte la question climatique. Et vice versa, les institutions climat ne s'intéressent pas, ou peu, aux questions de commerce ou de finance. Bien au contraire, puisque des «murs coupe-feu» ont été établis pour éviter que ces régimes interfèrent, comme le note le politiste Elmar Altvater $(2007,37)$. En excluant ces questions de la gouvernance climatique, on s'est ainsi privé de leviers importants pour infléchir la trajectoire d'émissions.

À la fois conséquence des blocages précités et facteur aggravant, une dernière dimension du schisme est temporelle. Elle réside dans le hiatus accablant entre la lenteur procédurale des 
négociations onusiennes et l'accélération des dynamiques qui ont transformé notre monde au cours des dernières décennies. Entre 1990 et 2011, le volume des flux marchands globaux a été multiplié par dix, avec des conséquences dramatiques pour l'évolution des émissions. La mondialisation économique s'est aussi accompagnée d'une croissance, inédite dans l'histoire, de l'économie chinoise, qui a atteint $10 \%$ annuels durant toute la décennie des années 2000. Comme cette croissance reposait entièrement sur les énergies fossiles, l'évolution des émissions chinoises a dépassé toutes les prévisions. Des bouleversements comparables - gaz de schiste, catastrophe nucléaire de Fukushima, essor des énergies renouvelables ont transformé le paysage énergétique mondial durant cette période. Face à ces dynamiques d'accélération dans différents domaines, les négociations climatiques ressemblaient de plus en plus à une «fabrique de la lenteur» (Aykut et Dahan, 2015, 395). L'exemple du protocole de Kyoto est instructif à cet égard. Alors que les bases des négociations pour le traité remontent au début des années 1990, son entrée en vigueur n'intervient qu'en 2005. Or, entre 1990 et 2005, le monde avait complètement changé : en 1990, la Chine était la $7^{\circledR}$ économie mondiale derrière l'Italie, alors qu'en 2005, elle était en phase de dépasser les États-Unis comme premier émetteur mondial de gaz à effet de serre. Prises entre scléroses institutionnelles et jeux d'ombre diplomatiques, les négociations climatiques poursuivaient alors inexorablement leur chemin, inaptes à prendre en compte cette évolution et à mesurer ses implications profondes sur la gouvernance du problème climatique.

\section{L'arène climatique, entre obésité et débordements}

Après Copenhague, les débats climatiques sont caractérisés par l'écart croissant entre un diagnostic scientifique de plus en plus alarmant - en apparence accepté par tous et l'insuffisance des efforts de réduction des émissions. Cet écart fait d'ailleurs l'objet d'un rapport annuel The Emissions 
Gap Report, publié depuis 2010 par le Programme des Nations unies pour l'environnement (UNEP, 2010). En réponse aux difficultés de l'arène climatique de prendre en compte les causes profondes de la croissance des émissions, on observe alors deux évolutions parallèles. D'une part, on charge la barque des négociations climatiques ${ }^{10}$. Si au début, celles-ci concernaient surtout la réduction des émissions et s'étendaient partiellement aux questions de financement et de transfert technologique, s'y s'ajoutent, au début des années 2000 , les problèmes d'adaptation et de vulnérabilité des populations face aux impacts du réchauffement. Les discussions sur le «mécanisme de développement propre» du protocole de Kyoto et l'initiative internationale en faveur du maintien des forêts, REDD (Reducing Emissions from Deforestation and Forest Degradation, 2008), — deux types de marchés de carbone créés pour permettre des investissements bas-carbone dans les pays du Sud - conduisent ensuite à l'inclusion de questions plus diverses, comme les technologies d'abattement des émissions, les pratiques d'usage des terres et la gestion des forêts. De manière un peu polémique, on pourrait dire qu'on négocie sur un ensemble croissant de sujets qui sont certes importants, mais qui ne visent pas le cœur du problème. En effet, on ne parle toujours pas, par exemple, de la régulation du commerce et des marchés énergétiques, ni de la coordination des efforts de transformation de la production énergétique, ni d'une transformation du système financier mondial qui alignerait celui-ci sur l'urgence climatique.

D'autre part, on observe des phénomènes de débordement, qui préparent une extension progressive du domaine de la gouvernance climatique. Ainsi, un nouveau type de campagnes d'activistes menés par des ONGs, think tanks et mouvements

10. La représentation graphique de l'évolution temporelle des thèmes de négociations dans Nicolas Baya-Laffite et Jean-Philippe Cointet (2016) illustre bien le phénomène. 
sociaux cible, au lieu de la seule réduction des émissions, les processus qu'ils estiment être à la source du problème. Ces acteurs commencent alors à s'intéresser, entre autres, aux flux financiers publics et privés qui soutiennent l'extraction pétrolière et charbonnière, et aux infrastructures qui perpétuent la dépendance aux énergies fossiles. Les organisations internationales deviennent une cible privilégiée de ces campagnes. Si la pression croissante de la société civile mondiale incite certaines de ces organisations à engager des réorientations stratégiques, celles-ci restent souvent partielles et n'ont qu'un impact marginal sur leurs activités. La Banque mondiale, par exemple, promeut des investissements dans les énergies renouvelables et fournit son expertise dans l'établissement de marchés de carbone (World Bank, 2014; World Bank et UNEP, 2014). Par ailleurs, elle souhaite devenir un acteur central de la finance climat en proposant de prendre en charge la gestion des fonds créés pour les pays en développement. Les chiffres de l'ONG Bank Information Center ${ }^{11}$ montrent cependant qu'elle continue à soutenir massivement la construction de centrales à charbon et d'infrastructures qui renforcent la dépendance aux énergies fossiles. De manière analogue, les chefs d'État réunis au sommet du G20 à Pittsburg, aux ÉtatsUnis en 2009, adoptent certes une résolution pour réduire les subventions aux énergies fossiles, qui débouche sur un effort inédit de monitoring et quantification de ces subventions (IEA et al., 2011). Les résultats concrets de l'initiative en termes de réduction des subventions sont cependant très limités et, de toute façon, l'enjeu central de ces réunions reste d'assurer la croissance de l'économie mondiale.

On retiendra donc de cette partie qu'une des forces motrices poussant à l'extension du domaine de la gouvernance climatique réside dans l'insatisfaction de tout un ensemble

11. <https://bankinformationcenter.org> (consulté le 02 juillet 2019). 
d'acteurs avec la manière dont la question climatique a historiquement été cadrée et prise en charge au sein des instances politiques compétentes. Ces acteurs posent un diagnostic commun : les négociations climatiques ne traitent pas du cœur du problème. Leur stratégie consiste donc à cibler d'autres arènes internationales, et à inciter les protagonistes de ces domaines à s'intéresser à l'enjeu climatique et à le prendre en compte dans leurs pratiques de gouvernance.

\section{Les sommets onusiens : des machines à climatiser}

Une autre réponse à la débâcle de Copenhague se prépare à l'intérieur du régime climatique. Les négociations reprennent en 2010 à Cancun et débouchent, l'année suivante à Durban en Afrique du Sud, sur l'adoption d'une nouvelle feuille de route pour une nouvelle tentative de trouver un successeur au protocole de Kyoto. L'année butoir de ce cycle de négociations est fixée pour 2015. Alors que les contours du nouveau traité restent encore flous, le gouvernement français propose d'organiser les pourparlers. La COP21, présentée comme un rendez-vous décisif pour la lutte contre le réchauffement global, se tiendra donc à Paris.

\section{La COP21 comme méga-évènement transnational}

En raison de son importance pour le futur de la gouvernance onusienne, la conférence présentait un intérêt évident pour nous chercheurs. Or c'était aussi un objet particulièrement complexe et difficile à aborder. Ceci d'abord en raison de sa taille : en plus des 30000 personnes officiellement accréditées par les Nations unies pour le centre de conférences au Bourget, plusieurs centaines de milliers de personnes du monde entier étaient attendues pour les manifestations, conférences et évènements qui se tiendraient un peu partout dans Paris. S'il est impossible d'appréhender un tel évènement dans sa totalité, nous avons mis en place un dispositif d'observation particulier afin de le couvrir le mieux possible. Ce dispositif 
réunissait une équipe d'une quinzaine de chercheuses et chercheurs autour d'une méthode originale «d'ethnographie collaborative», adaptée aux enjeux spécifiques que pose l'observation d'un "méga-évènement transnational» comme la COP21 (Campbell et al., 2014; Siméant et al., 2015). Une telle approche combine entretiens, observations et analyse de documents. L'intérêt est qu'elle permet d'étudier les différents espaces sociaux et ensembles d'activités qui composent un sommet onusien. En effet, selon la perspective adoptée, ces sommets apparaissent tantôt comme un marathon de négociations mettant en jeu des intérêts d'États et engageant des tractations diplomatiques dans des espaces souvent confidentiels, tantôt comme une foire ou un salon d'exposition, où ONGs, instituts de recherche et firmes présentent leurs idées et leurs technologies pour lutter contre le réchauffement, ou encore comme un happening populaire qui s'étale, sous forme de rassemblements, concerts et activités diverses, dans toute la ville. Notre objectif était de comprendre comment ces différentes facettes de l'évènement s'articulent entre elles, comment les acteurs d'une COP interagissent et forment des alliances à travers ces espaces et, plus généralement, quelles stratégies ils adoptent pour peser dans la gouvernance globale du climat.

Une autre difficulté liée à l'étude d'un tel sommet transnational consiste à déterminer ses limites temporelles (Schüssler et al., 2014). On ne comprend guère ce qui se passe durant les deux semaines d'une COP, si on ne prend pas en compte comment celle-ci a été préparée en amont. Ainsi, tout au long de l'année 2015, les délégations de pays se retrouvaient dans les sessions officielles de négociations intermédiaires, mais aussi lors de consultations bilatérales plus ou moins informelles, afin de se concerter et de sonder de possibles zones de compromis. Sans doute la plus importante de ces consultations a-t-elle eu lieu entre les États-Unis et la Chine en septembre 2015. Les présidents des deux pays ont alors rédigé une déclaration conjointe. Ils y faisaient part de leur volonté 
d'aboutir à un accord à Paris, tout en posant comme condition qu'un tel traité devait être fondé sur deux principes : d'une part, une approche volontaire, qui prévoit la soumission de «contributions nationales» dont le contenu serait librement déterminé par les États; d'autre part, l'adoption de la limite des $2^{\circ} \mathrm{C}$ comme objectif commun devant guider l'effort des pays. Bien avant la COP21, il était donc clair que les deux superpuissances du début du XxI siècle ne voulaient pas d'un accord qui leur imposerait des réductions contraignantes. Du côté de la société civile aussi, différents acteurs se concertaient, déterminaient leurs stratégies et essayaient de promouvoir leurs thèmes. Ainsi, une série d'opérations concertées visait, en mai 2015, les infrastructures de la production d'énergies fossiles. Des activistes ont alors investi des mines à charbon, interrompu l'approvisionnement de centrales thermiques et bloqué un port charbonnier en Australie. Organisée par une coalition de mouvements sociaux et d'ONGs d'environnement, la campagne Break Free visait à mettre l'extraction et la production d'énergies fossiles à l'agenda politique et médiatique de Paris. D'autres ONGs et des instituts de recherche se coordonnaient sur des thèmes plus proches du processus de négociations. Le World Resources Institute, par exemple, un think tank américain proche du parti démocrate, avait mis en place un cycle de consultations associant des représentants de quelques pays du Nord et du Sud, de think tanks et d'ONGs $^{12}$. L'objectif était d'esquisser les contours d'un futur accord qui serait aussi ambitieux que possible, tout en respectant les principales lignes rouges des États. Les consultations conclurent que l'accord devait fixer un objectif de température ambitieux, et combiner une approche volontaire pour les engagements des pays avec un cycle de réexamen régulier, qui permettrait de réévaluer ces engagements tous les cinq

12. Il s'agit de l'initiative ACT 2015 (Agreement for climate transformation ou Accord pour une transformation du climat). 
ans (Morgan et al., 2014). Il devait également accorder une place importante aux acteurs non-étatiques comme les villes et les entreprises, invitées à leur tour à soumettre des engagements volontaires. L'espoir était que cela permettrait de créer une «dynamique positive» pour le changement et de progressivement rehausser l'ambition. On note d'ailleurs une forme d'alignement discursif entre cette partie «réformiste» de la société civile mondiale et la frange la plus progressiste des associations d'entreprises. Un graphique récurrent dans les rapports de We Mean Business titré «Notre théorie du changement $t^{13}$ » illustre cet alignement. Il représente les mondes politique et économique comme deux sphères interdépendantes, qui forment une sorte de cercle vertueux. L'idée est que la transformation bas-carbone repose sur un jeu de vases communicants, où des dynamiques positives dans les deux sphères se renforcent mutuellement. L'enjeu de la gouvernance climatique serait donc de définir un objectif clair (les deux degrés) et de mettre en place un cadre réglementaire ambitieux - mais pas trop strict au début - qui stimulerait des innovations bas-carbone dans le monde économique. L'augmentation de la part des firmes qui investissent dans la transition libérerait en retour - ainsi va l'argument — de nouvelles marges de manœuvre dans le monde politique, grâce auxquelles de nouvelles régulations peuvent être introduites.

\section{La force d'attraction des conférences climatiques}

En somme, la période précédant la COP21 était marquée par une mobilisation remarquable, non seulement de la part des États mais également d'une diversité d'acteurs privés. Ces acteurs étaient aussi présents à la conférence elle-même, où ils s'employaient à peser sur les négociations et faire avancer leurs agendas. Leurs activités et interactions obéissaient à une logique spatiale particulière. Afin de ne pas s'y perdre, nous

13. <https://www.wemeanbusinesscoalition.org/about/> (consulté le 02 juillet 2019). 
avons distingué trois arènes, en nous servant d'une métaphore empruntée au festival d'Avignon (Dahan et al., 2009). En effet, à Paris comme à d'autres COPs, il y a d'abord le in c'est-à-dire l'espace onusien de la conférence. C'est l'arène des négociations, et l'activité principale dans cette arène est la production de textes. À la COP21, cette «zone bleue» (le bleu est la couleur de l'ONU) était localisée au centre de conférences au Bourget. On y voyait des délégués de pays assis autour de grands écrans montrant, sous forme de documents Word, les textes soumis à la discussion par les présidents de séance. Selon l'état d'avancement des pourparlers, certains passages de ces textes étaient colorés pour faciliter le travail de regroupement et de synthèse, ou mis entre crochets pour indiquer les formulations controversées et encourager la recherche de consensus. Outre les négociations, on peut assister à d'autres activités à une COP : les délégations de pays, les ONGs, instituts de recherche et firmes y sont présents, distribuent des rapports et organisaient des side-events. Dans cette arène du off, des représentants de la société civile institutionnalisée — c'est-à-dire accréditée par les Nations unies — s'engagent dans des activités de réseautage et de lobbying, échangent des idées et promeuvent leurs solutions. Le off fonctionne comme une sorte de caisse de résonnance, comme un «poumon» de la négociation, où un public averti suit et décrypte les négociations, et prend position sur des évolutions qui pourraient poser problème (Aykut et Dahan, 2015, 113). Au Bourget, tout cela se déroulait généralement dans la zone bleue mais en dehors des négociations, dans une ambiance de foire internationale. De manière sporadique, on y trouvait aussi de l'activisme et des mini-manifestations plus ou moins spontanées. Formellement interdites mais généralement tolérées, de telles rencontres étaient en règle générale organisées en réponse à des évolutions dans les négociations, par exemple quand des ONGs découvraient qu'une formulation à laquelle elles tenaient avait été enlevée ou mise entre crochets dans une 
nouvelle mouture du texte de négociations. L'objectif était alors de faire pression sur les négociateurs, en attirant l'attention du public et des médias présents dans la zone bleue sur leur cause. Une autre stratégie consistait à activer des contacts noués en amont avec des représentants étatiques spécifiques, prêts à porter leur cause à l'intérieur des négociations.

Enfin, le off du off se déroule généralement dans les rues, cafés et hôtels de la ville qui organise la conférence. C'est l'arène des actions médiatiques et évènements organisés à l'intention d'un public citoyen plus large, mais aussi des rencontres plus confidentielles sur des thématiques spécifiques. À la COP21, cela incluait deux espaces au Bourget, mais en dehors de la zone bleue : l'espace Générations climat dédié à la société civile, et la Galérie, un lieu d'exposition pour les entreprises. À ceci s'ajoutaient des activités diverses organisées dans Paris, comme des manifestations et concerts de rue, des expositions et conférences dans les grands hôtels, ainsi qu'un Village des alternatives à Montreuil. Le rapport aux négociations a pu être direct, par exemple quand des représentants de peuples autochtones manifestaient à Paris pour exiger, parmi d'autres demandes, l'inclusion d'une référence à leurs "savoirs traditionnels" dans le traité final. Il a pu aussi être plus indirect. Dans une action spectaculaire exécutée à l'aide de véhicules spécialement préparés et équipés, des militants de Greenpeace ont ainsi répandu une couleur jaune sur la place Charles-deGaulle et les avenues qui partent de son centre. Vu du ciel, l'espace coloré autour de l'Arc de Triomphe formait un soleil stylisé. L'ONG envoyait ainsi d'une part, un message aux diplomates réunis dans la zone bleue, appelés à s'accorder sur des mesures de soutien aux énergies renouvelables; d'autre part, l'action visait à capturer l'attention médiatique générée par la COP21 pour faire avancer, au-delà de l'arène climatique, une cause qui est traditionnellement au cœur de l'activisme de Greenpeace. 
Il se passait donc des choses très variées dans une multiplicité d'espaces durant les deux semaines de la conférence de Paris. En donnant lieu à une mobilisation sans précédent de la société civile, celle-ci a contribué à attirer tout un ensemble d'acteurs vers l'arène climatique. Selon que ceux-ci intervenaient dans la zone bleue ou dans des espaces plus périphériques de la COP21, ils se voyaient contraints de traduire leurs thèmes et intérêts en des termes plus ou moins proches de ceux qui prévalent dans les négociations climatiques. En retour, ces acteurs ont aussi influencé le débat médiatique, participé à la mise sur agenda de nouveaux enjeux et pesé sur le déroulement de la conférence et sur ses résultats. Ils ont en particulier, au moins en grande partie, soutenu l'émergence d'une nouvelle approche de la gouvernance, qui s'accommode du tournant opéré vers le volontariat et la soft law, tout en visant à compenser l'insuffisance des engagements des États par une association plus forte à l'effort de réduction des émissions des firmes et des entités infranationales. Comme nous allons le voir dans la prochaine section, cette nouvelle approche repose en grande partie sur l'espoir d'une mobilisation tous azimuts de la société civile mondiale.

\section{La climatisation comme stratégie de gouvernance}

L'adoption de l'accord de Paris ${ }^{14}$, à l'issue d'une année de tractations diplomatiques et deux semaines de négociations intenses, a été saluée par des observateurs du monde entier comme un grand succès. Quelles sont les grandes lignes du traité ? Au niveau substantiel, l'article 2 fixe d'abord un double objectif à long terme : contenir "l'élévation de la température moyenne de la planète nettement en dessous de $2^{\circ} \mathrm{C}[\ldots]$ en poursuivant l'action menée pour limiter les températures

14. L'accord de Paris et la décision de la COP qui l'accompagne peuvent être téléchargés sur le site $<$ https://unfccc.int $>$ (consulté le 02 juillet 2019). Pour des analyses détaillées du traité, voir Falkner (2016) et Bodansky (2016). 
à $1,5^{\circ} \mathrm{C} »$. La limite des $2^{\circ} \mathrm{C}$ est donc pour la première fois reconnue dans un traité international. Elle est assortie d'un objectif encore plus ambitieux de $1,5^{\circ} \mathrm{C}$, dont le statut juridique reste toutefois flou. Pour atteindre ces objectifs, l'accord appelle à un effort collectif, mais volontaire. Pour la première fois, cet effort inclut également les pays en développement. En contrepartie, le traité promet des aides financières pour la réduction des émissions et l'adaptation dans les pays en développement. S'il reste muet sur la hauteur exacte de cette aide, la décision de la COP qui l'accompagne fixe le chiffre de 100 milliards de dollars par an — et plus à partir de 2025. En revanche, ni la source de ces fonds, ni la forme sous laquelle ils doivent être mis à disposition — aide publique, prêts, investissements privés - ne sont précisées. Enfin, l'accord reconnaît le concept de "pertes et dommages», qui désigne des impacts du réchauffement comme la désertification ou la montée des mers, pour lesquels l'adaptation ne suffira pas. Comme la décision de la COP exclut explicitement toute responsabilité juridique des pollueurs pour de telles pertes, les effets concrets de cette notion potentiellement lourde de conséquences restent toutefois limités.

\section{Le pari d'une mobilisation de la société civile mondiale}

En somme, l'accord de Paris innove moins par ses dispositions substantielles, que par le cadre procédural qu'il institue. Ce cadre repose sur trois éléments : d'abord la soumission par tous les pays de plans climat nationaux nommés Nationally Determined Contributions ou NDCs, dont le contenu est librement déterminé par les États; ensuite, un «mécanisme de transparence» pour assurer la comparabilité de ces soumissions; enfin, une procédure de réexamen quinquennal, qui comprend un «bilan mondial», c'est-à-dire une discussion collective sur les efforts accomplis, qui précède de deux ans la re-soumission de nouveaux engagements. L'accord de Paris signe donc l'abandon définitif de l'approche contraignante du protocole 
de Kyoto et complète le tournant vers une gouvernance climatique par la soft law. Difficilement traduisible en français, ce terme renvoie à au moins trois réalités juridiques distinctes (Delmas-Marty, 2004) : l'accord est «flou» puisqu'il contient des formulations qui permettent différentes interprétations, notamment celles concernant les objectifs à long terme; il est «mou» car la plupart de ses articles sont formulés dans un langage non contraignant; enfin, il est «doux» puisqu'il ne prévoit pas de sanctions en cas de non atteinte des engagements des pays. Si cette souplesse a sans doute été nécessaire pour faire aboutir les négociations à la COP21, on peut se demander si elle ne compromet pas l'intégrité environnementale de l'accord, d'autant plus que les contributions nationales qui sont actuellement sur la table, ne suffisent de toute évidence pas à contenir le réchauffement à deux degrés ${ }^{15}$. Les architectes de l'accord répondent à cette question en renvoyant à l'outil de réexamen quinquennal des engagements. Celui-ci formerait le cœur d'une démarche inclusive combinant volontariat, construction de confiance et pression des pairs, qui doit être le vecteur d'un renforcement progressif des engagements. Il est intéressant de noter que l'idée d'une telle démarche cyclique a été introduite dans le débat, non par une délégation nationale, mais par l'initiative privée $A C T$ 2015, mentionnée ci-dessus. Le concept sous-jacent de dynamiques positives qui se renforceraient mutuellement fait aussi écho à la «théorie du changement» de We Mean Business. La société civile mondiale a donc joué un rôle important dans la mise en place de l'approche adoptée à Paris. Elle est également appelée à participer à sa mise en œuvre. D'une part, les analyses critiques d'ONGs et de think tanks sont considérées comme indispensables pour garantir la transparence du

15. Le site web <https://climateactiontracker.org > fournit une compilation constamment mise à jour des engagements des pays et des analyses sur leur effet prospectif sur le réchauffement futur. 
processus de réexamen et inciter les pays à rehausser progressivement l'ambition de leurs engagements (van Asselt, 2016). D'autre part, firmes, municipalités, régions et États fédérés sont encouragés à contribuer directement à l'effort de réduction en soumettant à leur tour des engagements volontaires. Enregistrés par le secrétariat de la Convention climat, ces plans climat sont ensuite mis à disposition du public sur un site internet créé à cet $\operatorname{effet}^{16}$. La publicité des promesses et la pression de la société civile doivent donc assurer la mise en œuvre des engagements volontaires d'acteurs publics, privés et infranationaux.

\section{Le narratif performatif d'une planète en transition}

Au-delà de sa dimension juridique, la gouvernance globale comporte aussi une dimension symbolique et discursive importante (Little, 1995). En effet, il est peu pertinent de réduire le résultat de la COP21 au document de 40 pages -18 pour l'accord et 22 pour la décision - adopté par les délégués nationaux. Les derniers jours de la conférence étaient aussi accompagnés d'une offensive médiatique des architectes de l'accord, qui vantaient inlassablement le momentum généré par la conférence et le signal économico-politique majeur envoyé par l'adoption de l'objectif des deux degrés. Cette campagne s'est poursuivie après la COP21, notamment par une mise en scène régulière des progrès réalisés à l'occasion des COPs ou de sommets extraordinaires convoqués entre ces rendez-vous annuels, comme le One Planet Summit à Boulogne-Billancourt le 12 décembre 2017. Ce travail discursif et symbolique enveloppe l'accord dans un récit téléologique, qui interprète la COP21 comme un tournant historique sur le chemin d'une transformation désormais devenue inéluctable de l'économie mondiale. En l'absence de toute contrainte ou possibilité de sanctions, cette narration fait partie intégrante de la nouvelle

16. $<$ https://climateaction.unfccc.int/> (consulté le 02 juillet 2019). 
gouvernance. En témoigne un remarquable entretien donné par une des architectes de l'accord, l'«ambassadrice chargée des négociations» à la COP21 Laurence Tubiana, peu après la conférence. Elle soutient dans les pages de Libération que le traité doit être «une prophétie auto-réalisatrice ${ }^{17} 》$. Là encore, l'argument repose sur l'imaginaire d'un cercle vertueux. Selon elle, le cap des $2^{\circ} \mathrm{C}$ doit aligner les attentes et visions du futur des décideurs politiques, chefs d'entreprise et investisseurs à travers le monde. Si une bonne partie parmi ceux-ci croit en l'imminence d'une transformation bas-carbone de l'économie mondiale et redirige ses décisions, activités et choix d'investissements en fonction de cette nouvelle donne, cela enclenchera une dynamique positive : un monde à $2^{\circ} \mathrm{C}$ deviendra alors plus probable, et de plus en plus d'autres acteurs suivront leur exemple. C'est cette mécanique qui doit permettre le respect de l'objectif de limitation de la température. L'approche de Paris ressemble donc à ce qu'on pourrait appeler une "gouvernance incantatoire» (Aykut, 2017), qui repose non pas sur la production de règles contraignantes, mais sur un processus cyclique et ritualisé qui mobilise promesses et signaux à l'intention d'une grande variété d'acteurs publics et privés à l'intérieur, mais aussi au-delà de l'arène climatique. Étant donné que la mobilisation tous azimuts de la société globale devient une stratégie délibérée et explicite pour atteindre les objectifs climatiques, la nouvelle gouvernance accorde une place de choix au processus de climatisation.

Le talon d'Achille d'une telle approche est la crédibilité des engagements à long terme. Pour parer au risque de démobilisation, les défenseurs de l'accord doivent donc assurer que le momentum qui soutient la performativité des objectifs soit

17. Losson C., «COP21 : L'accord doit être une prophétie autoréalisatrice». Libération, 17/12/2015, <https://www.liberation.fr/planete/2015/12/17/cop21-1-accord-doit-etre-une-prophetie-autorealisatrice_1421547> (consulté le 02 juillet 2019). 
continuellement entretenu et réactualisé. Le contrôle de la dimension discursive de la gouvernance — produire des récits, fournir des cadrages, imposer une interprétation - devient par conséquent un enjeu crucial. C'est dans ce contexte qu'il faut comprendre les réactions à la décision du président américain Donald Trump de quitter l'accord de Paris, comme le désormais célèbre «Make our planet great again» lancé par son homologue français Emmanuel Macron, et la réaffirmation quasi-rituelle de l'attachement des grandes puissances - Europe, Chine, Canada, Inde et autres - à l'accord de Paris. Ces réactions s'inscrivent non pas tellement, comme ce fut le cas après le retrait américain du protocole de Kyoto en 2001, dans une lutte pour la survie légale du traité. L'enjeu est plus symbolique que juridique : il s'agit de sauver le grand récit d'une «planète en transition», qui doit assurer la performativité de l'accord, et donc sa mise en œuvre.

\section{Ambiguiités et contradictions : le cas des politiques énergétiques}

De ce qui précède, on pourrait tirer la conclusion que l'extension de la question climatique vers d'autres domaines et d'autres arènes globales constitue un processus historique linéaire et inévitable, qui débouchera in fine sur une prise en charge plus efficace du problème climatique. La réalité est plus complexe. La climatisation décrit un processus incomplet et sélectif, aux conséquences parfois ambiguës ou contradictoires. Pour s'en convaincre, il suffit de s'intéresser de plus près à la manière dont l'enjeu climatique refaçonne les débats dans un domaine spécifique : la gouvernance des questions énergétiques (Aykut et Castro, 2017).

\section{Gouvernance de l'énergie :} une climatisation avant tout discursive

Comme environ deux tiers des émissions mondiales de $\mathrm{CO}_{2}$ proviennent de la combustion d'énergies fossiles, la question 
climatique est souvent présentée comme étant d'abord et surtout un problème de production énergétique. On sait aussi que pour rester sous la barre des deux degrés de réchauffement, il faudrait que les deux tiers des réserves d'énergies fossiles aujourd'hui connues restent inexploitées (McGlade et Ekins, 2015). Il n'est donc pas surprenant que les questions énergétiques - énergies fossiles et renouvelables en tête ont été très présentes dans le débat public en amont de la COP21. Ceci d'autant plus qu'on observe actuellement non pas un ralentissement des activités d'extraction et de production d'énergies fossiles, mais plutôt l'inverse : la course aux dernières ressources de pétrole, de charbon et gaz naturel fait rage. Elle est même accentuée par certains effets du réchauffement. Ainsi, la fonte des glaces en Arctique rendra accessibles d'importants gisements de pétrole et de gaz. Cette perspective aiguise déjà l'appétit des pays riverains, dont certains se positionnent pour pouvoir tirer profit, le moment venu, du dégel en cours.

La situation n'est guère plus encourageante du côté des organisations internationales mandatées pour s'occuper des questions énergétiques. L'Agence internationale de l'énergie (AIE), par exemple, qui fait autorité dans le domaine en compilant chaque année un volumineux état des lieux prospectif sur l'évolution de la demande et de l'offre énergétiques globaux, accorde certes une place de plus en plus importante aux questions climatiques. Sa publication phare World Energy Outlook inclut depuis 2008 un scénario «bas carbone». En même temps, l'organisation continue toutefois à encourager la mise en place de nouvelles infrastructures d'extraction, de transport et de combustion d'énergies fossiles. Qui plus est, la régulation des marchés énergétiques mondiaux est notoirement faible et parcellaire. Par conséquent, la question climatique ne joue qu'un rôle marginal dans le «grand jeu» de l'énergie, dominé depuis toujours par la poursuite d'intérêts géopolitiques et économiques. 
L'énergie constitue, en d'autres termes, l'exemple paradigmatique d'un domaine où le climat est à la fois omniprésent dans les discours politiques et médiatiques, et étrangement absent dans les pratiques économiques et de gouvernance.

Dans ce contexte, une série de campagnes et d'initiatives visaient à sortir de l'impasse politique en ciblant directement différents aspects de la question des énergies fossiles : la campagne Break Free de mai 2015 problématisait les activités d'extraction et de combustion; le mouvement Divest s'articulait autour du financement des infrastructures; le G20 mettait en exergue les subventions publiques à la production et la consommation; enfin, la Carbon Pricing Leadership Coalition proposait de réorienter les investissements par des taxes ou marchés de carbone.

\section{Gouvernance du climat : les enjeux énergétiques entre omniprésence et absence}

Les débats énergétiques étaient donc fortement «climatisés» avant la COP21. Qu'en est-il inversement : la question énergétique joue-t-elle un rôle dans les pourparlers climatiques? La réponse est négative. Quand on passe en revue les principaux traités de la gouvernance climatique - Convention climat, protocole de Kyoto, accord de Paris -, on note qu'il y est beaucoup question de réduction des émissions, mais très peu de politiques énergétiques. Dans l'accord de Paris, le mot «émission» (ou "émissions») apparaît 25 fois, tandis qu'«énergie» n'est mentionné qu'une seule fois, dans un passage sur le statut d'observateur de l'Agence internationale de l'énergie atomique. Le document ne contient donc aucune référence aux ressources et technologies énergétiques, à la question des subventions ou à des mesures concrètes pour réduire la part des fossiles dans le mix énergétique. Cette omission n'est pas un hasard, mais le résultat historique d'une stratégie employée par l'Arabie Saoudite et les principaux pays producteurs d'énergies fossiles, dont les États-Unis. 
Elle consiste à bloquer toute discussion explicite sur les politiques énergétiques sous prétexte que la Convention climat ne serait pas un traité sur l'énergie, mais un traité environnemental (Depledge, 2008). Alors que tout le monde semble s'accorder sur la nécessité d'une transformation profonde et rapide du système énergétique mondial, la gouvernance climatique reste donc muette sur les contours de cette transformation et les chemins pour y parvenir. L'initiative Yasuni ITT, portée par l'Équateur au tournant des années 2010, constitue une rare exception à cette règle. Le gouvernement équatorien proposait alors de renoncer à la mise en production d'un large gisement de pétrole découvert dans le sous-sol du plus grand parc national du pays en contrepartie de compensations financières. Face à l'insuffisance des promesses financières enregistrées quelques années après, l'initiative a échoué en 2013 (Damian, 2013).

Les discussions concernant l'article 4 de l'accord de Paris fournissent une autre illustration du statut ambigu des questions énergétiques dans le régime climatique. Elles permettent aussi de mieux comprendre les problèmes qui découlent de cette situation. Les diplomates étaient alors confrontés à la question épineuse d'une traduction de l'objectif des $2^{\circ} \mathrm{C}$ en des termes plus concrets, afin d'opérationnaliser l'objectif à long terme et de spécifier ses implications. Fallait-il déterminer une date précise pour la sortie des énergies fossiles, ou préconiser des mesures concrètes pour y parvenir? Ou suffirait-il de s'accorder sur la nécessité d'atteindre la «neutralité carbone» de l'économie mondiale le plus vite possible, laissant aux pays le soin de déterminer quand et par quels moyens ils comptent y arriver? Une proposition de compromis soutenue par un groupe de pays consistait à demander une «décarbonation» (ou «décarbonisation») de l'économie mondiale jusqu'à la moitié du XxI ${ }^{\mathrm{e}}$ siècle. Même si cette formule ne contient aucune référence à des ressources énergétiques précises, elle était de toute évidence toujours 
trop explicite pour certaines délégations nationales. Celles-ci faisaient valoir que parler de décarbonation reviendrait à condamner implicitement le recours aux énergies fossiles, ce qui serait hors du mandat de la Convention climat. En fin de compte, c'est donc l'objectif à long terme qui a été «décarboné». La mouture finale de l'article 4 stipule qu'il faut «parvenir à un équilibre entre les émissions anthropiques par les sources et les absorptions anthropiques par les puits de gaz à effet de serre au cours de la deuxième moitié du siècle». Cette formule alambiquée, d'apparence proche de l'objectif de décarbonation, renvoie en fait au concept de «zéro émissions nettes». L'idée consiste à compenser un dépassement temporaire de la trajectoire d'émissions compatible avec les $2^{\circ} \mathrm{C}$ par le recours futur à des technologies «d'émissions négatives» (Fuss et al., 2014). Ainsi, des centrales à biomasse couplées à des dispositifs de captage et de stockage de $\mathrm{CO}_{2}{ }^{18}$ permettraient en théorie de retirer du gaz carbonique de l'atmosphère, puisque le $\mathrm{CO}_{2}$ fixé par les plantes lors de leur phase de croissance est récupéré au moment de la combustion et injecté dans des réservoirs étanches dans le sous-sol. Les modélisations utilisées par le GIEC pour calculer les trajectoires d'émission en phase avec les objectifs de $2^{\circ} \mathrm{C}$ ou $1,5^{\circ} \mathrm{C}$ reposent aujourd'hui sur le pari d'une mobilisation massive de ces technologies dans les décennies à venir (Anderson, 2015). Le problème avec de tels calculs est qu'ils sont hautement spéculatifs. De fait, ils instaurent une "économie des promesses» (Joly, 2010), en soutenant l'illusion qu'une limitation du réchauffement à des niveaux à peu près tolérables resterait toujours possible, malgré la poursuite inchangée de modes de développement très carbonés. En dernière conséquence, cette forme de comptabilité créative permet de repousser toujours un peu plus loin la date définitive d'une sortie des fossiles.

18. Aussi appelés BECCS, pour bioenergy with carbon capture and storage. 


\section{Le prix de la climatisation : les sciences des sols à l'aune du climat}

La question des émissions négatives renvoie à un autre problème, dont je voudrais parler en conclusion : la climatisation de domaines ou spécialités académiques, ici des sciences des sols. Pour mettre en lumière les tenants et aboutissants de ce problème, je m'appuie sur une recherche empirique très fouillée d'une équipe de collègues sur la trajectoire politique et scientifique de l'initiative « 4 pour 1000 » (Kon Kam King et al., 2018; Fournil et al., 2018). De quoi s'agit-il? Ma première rencontre avec ce terme remonte à une conférence tenue au pavillon français durant la COP21. Devant une salle inhabituellement comble - le public se pressait le long des couloirs et à l'entrée - et en présence de nombreux journalistes, Laurence Tubiana et Stéphane Le Foll, alors ministre de l'Agriculture, présentaient cette initiative, dont je n'avais jamais entendu parler auparavant. Le cœur du propos était aussi simple qu'il semblait révolutionnaire : il suffirait d'accroître de seulement $0,4 \%$ les capacités de séquestration de carbone des sols à travers des pratiques agricoles et forestières adaptées, pour y stocker autant de $\mathrm{CO}_{2}$ que l'humanité émet chaque année. Boum! Quelle promesse!

\section{Une fenêtre d'opportunité pour promouvoir les recherches sur les sols}

L'initiative du «4 pour $1000 »$ ne venait pas de nulle part. Sa mise sur agenda politique résultait de la confluence, au cours des années précédant la COP21, de deux évolutions différentes, indépendantes dans un premier temps. La première était la montée en puissance progressive des enjeux liés à la dégradation et la gestion des sols au niveau des Nations unies. Ainsi, l'Organisation des Nations unies pour l'agriculture et l'alimentation (FAO) lança en 2012 le Global Soil Partnership associant organisations internationales et centres de recherche à travers le monde. Signe d'une structuration croissante de 
ces réseaux internationaux, un panel d'experts intergouvernemental fut établi l'année suivante, afin de compiler l'état des connaissances sur cette question et préparer des initiatives politiques. La mobilisation de la communauté des sols conduisit finalement à la proclamation d'une «année internationale des sols» en 2015. La question des sols était donc très présente dans les milieux onusiens quand les préparatifs pour la conférence de Paris commencèrent. La deuxième évolution concerne un début de réflexion sur les liens entre sols et changement climatique. En effet, depuis un peu plus d'une décennie, des travaux en sciences des sols et agronomie avaient tenté d'évaluer l'impact de la gestion des sols sur le réchauffement en cours. Ces recherches s'intéressaient aux flux entre le carbone terrestre et le carbone atmosphérique, qui peuvent être positifs quand du carbone est fixé dans les sols ou la biomasse, ou négatifs quand la décomposition de matière végétale ou des changements d'usage des terres conduisent au relâchement de $\mathrm{CO}_{2}$. Le chiffre de $0,4 \%$ fut établi dans ce cadre, dans une note de 1999 pour l'Académie d'agriculture de France, comme une première estimation très approximative de l'impact potentiel de différentes pratiques de gestion des terres (Arrouays et al., 1999). Ces travaux restaient toutefois assez confidentiels dans un premier moment. Au début de l'année 2015, ni la question de la contribution potentielle des sols à la réduction des émissions, ni le chiffre de $0,4 \%$ n'avaient réellement percé dans les milieux d'experts du climat ou dans le débat politique.

La réémergence de ce chiffre plus de quinze ans plus tard fut le fruit d'un concours de circonstances spécifiques, grâce auxquelles la COP21 devint comme une fenêtre d'opportunité inédite pour la mise sur agenda de la question des sols dans l'arène climatique. Du côté des scientifiques travaillant dans le domaine, le sentiment général était alors celui d'une marginalisation croissante, qui se reflétait notamment dans un amenuisement progressif des financements publics 
pour la recherche. Dans ce contexte, l'attention politique et médiatique générée par la conférence de Paris représentait pour ces scientifiques une occasion bienvenue pour donner une visibilité internationale à leur domaine. Afin de se positionner en interlocuteur légitime dans le débat climatique, certains d'entre eux mettaient en avant la contribution potentielle d'une meilleure gestion des sols à la lutte contre le réchauffement global, en utilisant le chiffre approximatif mais simple à communiquer du « 4 pour $1000 »$. Du côté du régime climatique, ce narratif s'insérait parfaitement dans l'esprit de la nouvelle gouvernance mise en place à Paris. Le discours positif promu par les organisateurs de la conférence consistait à mettre en avant les solutions au lieu d'insister sur les problèmes. En s'intéressant aux sols comme des puits de carbone plutôt que de les considérer d'abord sous l'aspect de leur contribution historique au réchauffement, comme ce fut le cas auparavant, l'initiative du « 4 pour $1000 »$ était donc en phase avec ce discours. Par ailleurs, l'argument sur le stockage du carbone dans les sols s'articulait parfaitement avec l'essor récent du débat sur les émissions négatives dans l'arène climatique, et plus généralement avec le biais persistant favorable aux solutions techniques.

\section{Étudier les sols à travers un prisme climatique}

L'exemple des «4 pour $1000 »$ illustre donc parfaitement comment s'exerce la force d'attraction du régime climatique et les processus sociaux à travers lesquels elle se déploie. Il permet aussi d'en mieux saisir les conséquences. Dans le cas des sciences des sols, la COP21 a d'abord conduit à un processus d'institutionnalisation, avec la création d'une série d'initiatives, de réseaux et de programmes de recherche tels que l'International Soil Carbon Network (ISCN) basé aux États-Unis, le collectif français Carbone des Sols, Mécanismes de Stabilisation (CarboSMS), ou encore le Soil-Net project soutenu par la Société britannique des sciences des sols. 
La visibilité accrue du domaine a aussi eu comme corollaire un accroissement des financements de recherche. Or cet accroissement ne concerne pas toute la gamme des recherches menées en sciences des sols. La capacité d'appliquer un cadrage "climat» à ses recherches constitue désormais un atout important - selon certains scientifiques, un véritable point de passage obligé - pour obtenir des fonds. Qui plus est, la formule du « 4 pour $1000 »$ est devenue une notion-clef dans les appels à projets en sciences des sols. On retrouve ici une mécanique commune dans beaucoup de domaines climatisés : à l'instar des objectifs de température $-2^{\circ} \mathrm{C}$, puis $1,5^{\circ} \mathrm{C}$ - dans les recherches en modélisation énergétique et climatique (Guillemot, 2017), des notions ou chiffres spécifiques circulent entre débat expert et débat politique. Quand ils finissent par s'imposer dans l'arène de gouvernance, ces notions ou chiffres viennent ensuite structurer les programmes de recherche, contribuant ainsi à légitimer certains objets et favoriser certaines approches, au dépens d'autres.

Cette observation me permet d'insister sur un dernier point. Pour beaucoup de domaines scientifiques ou politiques en quête de reconnaissance, se rattacher à l'arène climatique est attractif parce que celle-ci offre des opportunités en termes de visibilité, de prestige et de financements. Toutefois, ce rapprochement a un prix, puisque la climatisation transforme les domaines concernés. Là encore, l'exemple de l'initiative du «4 pour 1000 » est édifiant. D'abord, si la formule a pu s'imposer à Paris, c'est qu'elle formule le problème en termes de carbone. Le carbone est la lingua franca du régime climatique, du moins pour ce qui est des débats sur la réduction des émissions. Afin d'être entendu dans cette arène, il faut donc être en mesure de traduire son objet ou sa thématique en ces termes. On comprend aisément que cette réduction de la complexité d'un enjeu donné à cette seule et unique dimension peut avoir des effets hautement problématiques. La prédominance des nombres et de la quantification est une deuxième caractéristique du régime 
climatique, qui affecte les domaines climatisés. Le succès de l'initiative s'explique donc aussi par le fait qu'elle propose d'emblée une entrée par des chiffres. L'envers de la médaille est que ce biais s'accompagne souvent d'une marginalisation d'approches scientifiques et politiques qui résistent à une traduction en des grandeurs facilement mesurables et quantifiables. Cela est étroitement lié à un troisième point, à savoir la centralité de la modélisation numérique dans la production de connaissances (Dahan, 2010; Edwards, 2010). De nouveaux entrants dans l'expertise pour la gouvernance climatique se voient donc souvent cantonnés à un rôle plutôt auxiliaire au début, consistant à produire, soit de nouveaux jeux de données pour affiner des simulations climatiques, énergétiques ou économiques, soit des formalisations qui permettent d'élargir le périmètre d'un modèle existant en décrivant un processus ou un phénomène nouveau. Cela conduit à privilégier des approches visant à identifier des processus généraux et invariables, susceptibles de faire l'objet d'abstractions qui peuvent être incluses dans le formalisme des modèles dominants. Dans le cas des sciences des sols, l'entrée dans l'expertise climatique s'est donc faite aux dépens de recherches plus qualitatives, qui ont pour objet non pas d'établir des abstractions mathématiques, mais de retracer une histoire contextualisée, située et dynamique des sols. Une quatrième caractéristique est l'entrée de nouveaux acteurs dans les domaines climatisés. Cette entrée est souvent motivée par la difficulté qu'éprouvent les modélisateurs à articuler les approches qui prévalent dans les domaines en question avec leurs pratiques de modélisation. Ainsi, les chercheurs en sciences des sols ont dû constater que des physiciens-modélisateurs commençaient à produire eux-mêmes les formalisations simples dont ils avaient besoin pour représenter la dynamique des sols dans leurs simulations. En se substituant ainsi aux spécialistes du domaine, ces nouveaux experts accentuent encore la tendance à la marginalisation, au sein même des sciences des sols, de certaines approches et objets. 
Cinquième point, pour être entendu dans l'arène climatique, il faut désormais adopter un discours positif. Les recherches sur les sols ne constituent pas une exception à cette règle. Historiquement, celles-ci adoptaient une vision dynamique des sols, insistant autant sur leur capacité à séquestrer du carbone qu'à en relâcher, en fonction de processus naturels et de changements dans les pratiques et l'usage des terres. Depuis leur entrée dans l'arène climatique, le centre de gravité de ces recherches s'est progressivement déplacé, en faveur de la fonction positive des sols comme puits de carbone. L'accent est donc mis en priorité sur l'étude des mécanismes qui déterminent les capacités de séquestration des sols, et sur les pratiques de gestion à mêmes d'augmenter ces capacités. Enfin, une dernière spécificité de l'arène climatique est la mise en avant de solutions techniques. L'innovation technologique et l'économie de marché sont considérées comme les principaux vecteurs d'une transformation bas-carbone, tandis que la question des changements sociaux et politiques nécessaires pour opérer une telle transformation ne trouve que peu d'échos. Ainsi, l'initiative du «4 pour $1000 »$ avait au début donné lieu à une réflexion sur des pratiques agricoles plus durables et adaptées aux conditions locales. Ces débats se sont progressivement cristallisés autour de la notion d'agroécologie, un terme polysémique pouvant autant renvoyer à une agriculture écologique et paysanne, qu'à des pratiques hautement technicisées d'une agriculture sans labour. En germe, on voit ici le risque d'un glissement vers des enjeux très classiques d'augmentation du rendement agricole ${ }^{19}$. Dans les recherches sur les sols comme dans d'autres domaines,

19. Ceci d'autant plus que les questions de productivité occupent une place de choix dans la définition de «l'ambition de l'initiative» sur le site internet qui lui est consacré, à savoir « inciter les acteurs à s'engager dans une transition vers une agriculture productive, hautement résiliente, fondée sur une gestion adaptée des terres et des sols, créatrice d'emplois et de revenus et ainsi porteuse de développement durable», <https://www.4p1000.org/fr> (consulté le 05 octobre 2019). 
le rapprochement avec l'arène climatique a donc conduit à une reformulation des enjeux liés aux interactions complexes entre dynamiques socioéconomiques et processus naturels en des termes compatibles avec le cadrage dominant dans la gouvernance climatique : réduction de la question des sols à leur seule capacité à fixer du carbone; quantification de cette capacité et identification de processus généraux permettant de modéliser les processus sous-jacents; enfin, évaluation de solutions techniques permettant de maximiser l'apport de ces processus à la réduction des émissions.

\section{Conclusion}

Je voudrais conclure en revenant à la question, posée en introduction, du sens à donner au phénomène de climatisation : de quoi est-il le nom, et quel peut être son rôle dans la lutte pour contenir le réchauffement et gérer ses effets? La réponse est double, et en partie contradictoire : l'extension du domaine de la gouvernance climatique apparaît d'une part comme un processus historique salutaire et, en dernière conséquence, inévitable. Salutaire dans la mesure où il témoigne d'une prise de conscience croissante de la transversalité du problème climatique, qui met en jeu les fondements de notre modernité industrielle, du consumérisme des sociétés du Nord à l'extractivisme des pays du Sud, de l'organisation libérale et capitaliste de l'économie mondiale à la structure d'un système international basé sur la concurrence entre États souverains. Inévitable, puisqu'il paraît illusoire de vouloir traiter un tel problème dans le seul cadre du régime climatique onusien. Le processus de climatisation signe donc notre entrée dans une nouvelle condition humaine, dans laquelle le réchauffement provoqué par nos activités et les efforts pour le contenir affecteront progressivement tous les domaines de nos sociétés, en s'immisçant dans les débats politiques et les luttes sociales autant que dans les activités des firmes et les routines de gestion administratives. D'autre part, le processus 
de climatisation se déploie actuellement selon des modalités qui sont profondément problématiques. Son intensification récente résulte de l'échec historique de la gouvernance globale à prendre la mesure du problème et à l'endiguer; échec qui s'est nourri d'un cadrage trop étroit de la question en termes d'émissions et de solutions techniques. La climatisation tend à étendre ce cadrage à d'autres domaines. Elle s'accompagne donc souvent d'une marginalisation des réflexions portant sur les dynamiques sociales et politiques qui perpétuent des modes de vie et de développement insoutenables. L'incapacité à pointer les paradoxes et contradictions au cœur de la gouvernance climatique fragilise à son tour toute tentative de proposer une vision cohérente de la profonde transformation économique et technique, mais aussi sociétale et politique, nécessaire pour faire face à la dégradation du climat. En somme, le processus de climatisation apparaît donc, à lui seul, comme insuffisant pour inverser les tendances actuelles d'un accroissement quasi incontrôlé des émissions mondiales et d'une gestion peu solidaire des impacts du réchauffement en cours. Pour avoir une chance d'inverser ces tendances, l'extension du domaine de la gouvernance climatique doit se doubler d'une dynamique inverse, consistant à changer les rapports de force et redéfinir les termes du débat dans l'arène climatique. Cela passera nécessairement par une intensification des initiatives, campagnes et mouvements divers, à travers lesquels une large panoplie d'acteurs tente actuellement d'imposer d'autres cadrages dans le débat climatique, mais aussi au-delà, dans les autres domaines attirés dans le giron de la gouvernance climatique. 


\section{Discussion}

Question - J'ai compris grâce à votre exposé, toute la lourdeur, la lenteur, et aussi la fragilité de la gouvernance multilatérale au niveau des États. Fragilité puisqu'on voit que des États peuvent quitter ce qu'ils ont signé la veille. Ce qui m'a paru tout aussi intéressant dans votre exposé, c'est l'émergence d'autres types d'initiatives, par les entreprises, les villes. Comment voyez-vous l'équilibre à l'avenir entre ces différents types d'acteurs dans cette gouvernance mondiale? Le monde de l'entreprise, ou les collectivités locales peuvent-ils aller plus vite et plus loin que les États? Est-ce que ce sont eux qui vont sauver la planète?

Stefan C. Aykut - L'entrée de nouveaux acteurs dans la gouvernance climatique soulève effectivement des questions intéressantes. On peut en avoir une vision enchantée, et se réjouir du fait que des entreprises, des collectivités locales et des régions fassent, elles aussi, des efforts. Qu'elles s'intéressent à l'empreinte carbone de leurs activités et élaborent des plans pour la réduire. Cela est significatif dans la mesure où les organisations bureaucratiques, qu'elles soient publiques ou privées d'ailleurs, n'agissent en règle générale que sur ce qu'elles prennent en compte dans leurs bilans comptables. L'établissement de comptabilités carbone dans ces organisations est donc en soi une évolution positive. Cela dit, je pense aussi que les effets concrets de cette évolution resteront très limités tant que l'on ne touche pas plus fondamentalement aux structures des marchés, et aux incitations perverses que ceux-ci continuent à donner aux acteurs économiques. Tant qu'on n'active pas les leviers disponibles pour changer cette situation, il restera très difficile pour une entreprise de prendre les «bonnes» décisions. Il n'y a pas beaucoup d'entreprises qui peuvent se permettre d'investir 
dans les énergies renouvelables si le charbon est plus profitable, ou de changer leur approvisionnement énergétique si les énergies carbonées dominent le réseau électrique. Une multinationale exposée à la concurrence internationale et au dictat d'une profitabilité sur le court-terme des marchés financiers ne sera jamais environmental friendly si cela n'est pas au cœur de son modèle économique. En somme, ces initiatives sont certes encourageantes et méritent d'être soutenues, mais elles ne peuvent pas se substituer à l'action politique. Nous avons également besoin de régulations plus strictes, de taxes écologiques et de choix stratégiques pour réorienter les investissements publics. Il s'agit de redéfinir le cadre de l'activité économique, au niveau national comme à l'échelle internationale. Étant donné l'aggravation prévisible de la situation dans les décennies à venir, ces choix seront faits tôt ou tard, et ces régulations viendront, sous une forme ou sous une autre. Mais cela risque de se faire à un moment où il sera déjà trop tard pour opérer une transition à peu près ordonnée, à même de prévenir les pires conséquences du réchauffement tout en préservant les acquis sociaux et démocratiques de notre civilisation thermo-fossiliste. Il est donc primordial de prendre les «bonnes» décisions sans plus attendre. Un exemple, tiré de l'ouvrage Climate Change in World Politics du politiste John Vogler (2016) : au milieu des années 1990, les négociations climatiques se sont progressivement focalisées sur la répartition de l'effort de réduction en fixant des quotas d'émissions aux pays développés. Que se serait-il passé si, au lieu de cette approche, on s'était mis d'accord sur l'introduction d'une taxe, même assez modeste au début, sur l'extraction et le commerce du charbon? L'effet en termes de réduction des émissions aurait sans doute été beaucoup plus important. Les leviers politiques existent. Si une série de pays se mettait d'accord sur le principe d'une sortie du charbon à un horizon pas trop lointain, et que ces pays s'efforçaient de coordonner leurs efforts en introduisant 
des régulations de l'extraction et du commerce de charbon, cela changerait la donne pour beaucoup d'acteurs économiques. Même si certains pays continuaient à utiliser cette source d'énergie pour plus longtemps encore, le cap serait fixé. Le marché mondial des énergies fossiles doit être régulé. C'est inévitable! On ne peut pas engager une transformation aussi profonde de nos économies uniquement par des mesures «en fin de tuyaux» et par la voie du volontariat.

Question - Vous avez dit que parler de changement climatique, c'est "parler carbone». Oui, mais on peut aussi parler méthane. J'aurais aimé que vous précisiez un peu cet aspect parce que ce ne sont pas les mêmes réseaux qui sont concernés.

Stefan C. Aykut - Je suis entièrement d'accord sur le fait qu'il faudrait parler davantage du méthane. Le sujet n'est d'ailleurs pas absent des négociations. Le protocole de Kyoto, par exemple, porte sur un «bouquet» de 6 gaz à effet de serre, dont le méthane. Une des difficultés qui compliquent une meilleure prise en compte du méthane dans la gouvernance climatique est que la contribution relative de ce gaz au réchauffement - calculé à l'aide de son «potentiel de réchauffement global» par rapport au $\mathrm{CO}_{2}$ - dépend beaucoup de l'échelle temporelle à laquelle on se situe. Par convention, on considère généralement un horizon de 100 ans. À cet horizon, une masse donnée de méthane contribue 25 fois plus au réchauffement que la même masse de $\mathrm{CO}_{2}$. Le problème est que ce chiffre augmente considérablement quand on considère des durées plus courtes. À 20 ans par exemple, on passe à un potentiel de réchauffement de 72 ! On pourrait donc obtenir des résultats importants sur le court terme, si on agissait davantage sur la réduction des émissions de méthane. Pourquoi ne le fait-on pas, ou pas suffisamment? À mon avis, cela tient d'abord à une forme de dépendance au sentier de la gouvernance climatique, qui provient de la façon dont les débats ont été cadrés et dont les négociations 
se sont structurées. La centralité du carbone remonte au tout début de la mise sur agenda du problème : dans les années 1970 et 1980, les rapports d'expertise américains utilisaient le terme du $\mathrm{CO}_{2}$-problem pour désigner la question climatique. Ensuite, la question du méthane soulève aussi des enjeux spécifiques, qui ont historiquement entravé sa prise en charge politique. Dans la plupart des pays non-gaziers, l'élevage de ruminants et l'usage des terres constituent les principales sources de méthane. Les grandes rizières, par exemple, en émettent beaucoup. Or les questions d'agriculture et d'alimentation sont politiquement très sensibles. Les quelques tentatives de les mettre à l'agenda des négociations climat se sont heurtées à une forte résistance, venant principalement des pays du Sud, qui y voyaient une mise en cause de leur souveraineté nationale. La situation n'est guère plus encourageante au niveau national. L'exemple du Brésil est intéressant dans ce contexte : l'élevage bovin y constitue, selon la période considérée, soit la première, soit la deuxième source d'émissions de gaz à effet de serre. L'ordre retenu est finalement peu important, puisque la pression sur les terres exercée par l'élevage est considérée comme le vecteur principal de l'autre grande source d'émissions, la déforestation. On pourrait donc s'attendre à ce que les questions d'alimentation et d'élevage soient au centre du débat public brésilien sur les politiques climatiques. Or il n'en est rien, comme le montre une étude sociologique récente (Lahsen, 2017). Au lieu de s'intéresser au rôle de l'élevage et de la production de viande dans l'évolution des émissions brésiliennes, les médias du pays focalisent sur la production énergétique, les voitures à éthanol ou la déforestation. Bien entendu, le Brésil constitue un cas extrême et les ordres de grandeur sont différents dans d'autres pays. On observe néanmoins des dynamiques assez similaires conduisant à la marginalisation des enjeux liés à l'agriculture et à l'alimentation dans le débat public français ou allemand. 
Question - Sur la question du méthane, il me semble que l'on est en train d'assister à une évolution. On parle maintenant partout de sustainable diets, dans les organisations internationales, au niveau européen et dans les prospectives qui sont en train de se mettre en place. Je crois qu'il y a peu (guère plus d'un an), on considérait le régime alimentaire comme une chose sur laquelle on n'avait aucune prise. C'était un état de fait que l'on ne pouvait pas changer : il fallait s'accommoder des conséquences de ce que les populations demandaient à manger. J'ai l'impression que tout récemment, le concept de sustainable diets est devenu une variable sur laquelle on peut avoir une prise. On parle ainsi d' "évolution des régimes alimentaires ». J'ai l'impression qu'on assiste à un basculement et que l'on passe justement d'un moment où l'on considérait que c'était un facteur culturel sur lequel il était inapproprié de vouloir discuter, à sa prise en compte au même titre que les autres paramètres. On n'est peut-être pas plus volontariste pour se mobiliser à son sujet que sur celui de l'énergie, mais au moins la nécessité d'un changement dans nos habitudes alimentaires est maintenant dans le spectre des paramètres qui sont entre nos mains.

Stefan C. Aykut - Je vous rejoins sur le constat d'une certaine montée en puissance de ces thèmes depuis quelques années. C'est notamment dû au travail d'expertise et de mobilisation d'une série d'ONGs, qui essaient de faire la jonction entre la lutte contre le réchauffement d'une part, et les questions relatives au végétarisme et au bien-être animal de l'autre. Mais en fin du compte, cela reste quand même assez marginal dans les débats publics nationaux et la gouvernance globale. Il me semble par ailleurs qu'on peut rapprocher les problèmes associés à la réduction des émissions de l'élevage à ceux qu'on observe dans un autre secteur, celui des transports. Dans les deux cas, on peut, jusqu'à un certain point, opérer des réductions au niveau des producteurs, par exemple à travers des régulations qui visent à améliorer l'efficacité des véhicules 
ou à optimiser la gestion des fermes. En revanche, il apparaît beaucoup plus difficile d'agir de la même manière, d'utiliser les mêmes instruments, pour s'attaquer au niveau individuel. Tenter de réguler les régimes alimentaires ou le transport individuel, c'est soulever des questions qui touchent au cœur de nos modes de vie. Pour l'instant, il n'y a pas beaucoup d'hommes et de femmes politiques prêts à tester jusqu'où leur électorat accepterait des régulations sur ses modes de consommation. Par conséquent, ces questions sont rarement traitées autrement qu'à travers des campagnes d'information et de sensibilisation. J'ai l'impression qu'en Allemagne, par exemple, il sera finalement plus facile de sortir du charbon dans un futur pas trop lointain, que de vraiment s'attaquer à la question du transport individuel, tant qu'il n'existe pas d'alternative technologique prête à l'emploi et à peu près équivalente en termes de confort. Et je pense que cette difficulté tient au moins autant à la peur de s'immiscer dans la vie privée des électeurs, qu'à l'influence du lobby des constructeurs. C'est en tout cas mon sentiment, mais je peux me tromper.

Question - Je voudrais revenir sur l'émergence des nouveaux acteurs, en particulier les entreprises. Effectivement, je suis assez peu partisan de cette vision enchantée dans laquelle les nouveaux acteurs viendraient prendre en charge la question climatique. Je suis assez d'accord avec le fait qu'une entreprise ira toujours au moins coûteux pour ses activités, et donc que le capitalisme ne peut pas être spontanément régulateur du climat. Donc, il y a quelque chose que je n'ai pas compris pendant les négociations. Á un moment, on a vu qu'il y avait une question de rapport de forces entre les entreprises et les États, qui s'étaient focalisés autour de la taxation. Effectivement, on est sorti du monde de Kyoto où l'on pensait imposer des règles, mais il me semble qu'en amont de la COP21, il y a eu un moment où l'on a beaucoup négocié, parlé de la solution incitative qui permettrait de donner un cadre à tout le monde et à toutes les entreprises. Il s'agissait de définir 
au moins un prix du carbone ou peut-être des instruments de taxation. Et finalement, j'ai l'impression que cela a disparu, et je n'ai pas très bien compris dans quelles circonstances. J'aimerais avoir des éclaircissements là-dessus.

Stefan C. Aykut - Il y avait en effet, en amont et pendant la COP21, une importante mobilisation internationale sur ce thème. La Carbon Pricing Leadership Coalition, par exemple, regroupait des organisations internationales et des gouvernements, dont ceux de l'Allemagne et de la France, mais aussi, chose remarquable, toute une série de grandes firmes multinationales. Pour ratisser large, la coalition ne préconisait pas un instrument en particulier mais centrait son discours sur la nécessité de «mettre un prix» aux émissions de $\mathrm{CO}_{2}$, que ce soit à travers des taxes, des marchés d'émissions ou des systèmes hybrides. Comment apprécier la portée de cette initiative? Trois remarques sur ce point. La première est que l'existence même de cette coalition signale sans aucun doute des recompositions en cours dans une partie des milieux économiques. Pour s'en convaincre, il est utile de se rappeler des débats autour de l'introduction d'une taxe carbone européenne dans les années 1990. Une première proposition en ce sens, portée par la Commission européenne avant la conférence de Rio en 1992, avait rencontré une résistance féroce des lobbies industriels européens, qui réclamaient son retrait pur et simple. Ils ont obtenu gain de cause, non seulement avant Rio, mais aussi lors d'un épisode similaire quelques années plus tard, au moment des préparatifs pour la conférence de Kyoto. Certaines des firmes qui étaient montées au créneau à l'époque — pas toutes — ont de toute évidence revu leur position depuis. Comment expliquer ce revirement? Je pense qu'il y a d'abord une forme de prise de conscience. Une partie des milieux de l'entreprise se rend peu à peu à l'évidence qu'il ne leur sera pas possible de retarder infiniment des mesures plus volontaristes pour réduire les émissions. Parmi ces mesures, les firmes ont surtout peur d'une régulation 
stricte par des standards, normes d'émission ou fermetures administratives. Leur stratégie consiste donc à tenter d'influer sur le processus politique, afin que la décarbonation se fasse au rythme et dans des conditions qui leur conviennent. Assez naturellement, elles préfèrent les mécanismes de prix - taxes ou marchés de permis - à la régulation directe. La deuxième remarque concerne le contenu de la proposition de la coalition. L'initiative promeut une approche décentralisée, où différents systèmes et prix coexisteraient. À la différence d'initiatives antérieures, elle n'exige donc pas un prix carbone unique au niveau global. Elle se démarque ainsi du mantra de certains économistes, qui continuent à rêver d'un système homogène au niveau global. Quand on est un peu au fait de l'histoire des négociations, on sait très bien qu'une telle idée n'a aucune chance d'aboutir dans un futur proche. Un prix unique n'est pas acceptable pour l'Inde, la Chine et d'autres pays du Sud. Pourquoi devraient-ils appliquer le même prix que les pays développés, alors que proportionnellement, celui-ci toucherait leurs populations beaucoup plus durement? À l'instar de ce qui se passe actuellement en Chine, ces pays peuvent et vont, au moment qui leur semble opportun, mettre en place des systèmes qui leur conviennent. La dernière remarque concerne le statut de cette question dans les négociations. L'idée de fonder la lutte contre le changement climatique sur une coordination par les taxes, au lieu de fixer des objectifs de réduction, était promue par certains pays au tout début des négociations. La plupart des firmes pourraient très bien s'accommoder d'une telle solution : un prix carbone uniforme au niveau mondial créerait, selon le terme consacré, un level playing field, puisqu'il augmenterait les coûts de production de tous les compétiteurs dans un secteur donné de la même manière. Cette idée a disparu des négociations climat pour une raison très simple, qui est la même que pour les questions d'énergie : elle ne convient pas aux pays exportateurs de pétrole, de charbon et de gaz. Par 
le passé, toute discussion à ce sujet a systématiquement été bloquée par ces pays. Encore aujourd'hui, ils ne veulent pas entendre parler d'un prix carbone dans les négociations.

Question - Je suis médusé. Dans votre exposé, ces mégaévènements comme les COPs constituent une espèce de grand élan vers une sorte de gouvernement mondial, Vous parlez de gouvernance du climat, de climatisation. Je me demande comment cet élan arrive à réunir tout le monde alors que l'on n'arrivera pas à résoudre le problème dans lequel nous nous sommes mis. Une autre chose me frappe : j'ai l'impression que l'expertise scientifique a eu pour seul effet de déclarer qu'il y avait un risque climatique très important. Et ensuite, toutes les négociations, toutes les tentatives semblent être très indépendantes de ce que dit l'expertise du GIEC. On ne lui en demande peut-être pas plus, par exemple un diagnostic des solutions possibles. Donc je me dis que les " 4 pour 1000 » ont eu au moins pour mérite que les experts poursuivent les débats. Et c'est un point intéressant car le problème me semble venir de la faible liaison entre les expertises et toute la partie politique globale.

Stefan C. Aykut - Je comprends qu'on puisse être médusé par cette gouvernance, par la taille des conférences onusiennes et la complexité des négociations, et aussi par le contraste saisissant entre la lenteur du processus et l'urgence climatique. Des spécialistes des relations internationales, qui ont une vision classique du système international comme espace anarchique dominé par le seul jeu d'intérêts des États, vont d'ailleurs généralement mettre en avant qu'au-delà des insuffisances de la gouvernance globale, le fait même que les pays se rencontrent et qu'ils créent des institutions est remarquable. On peut avoir une telle vision des choses. Elle n'est pas fausse, puisque les pays qui se rencontrent à ces conférences se livrent en parallèle à une concurrence féroce pour des parts de marché et l'accès aux ressources, fossiles et autres. Parmi 
ces pays, certains n'entretiennent même pas de relations diplomatiques. D'autres sont officiellement en guerre, latente ou ouverte. Et malgré tout, ces pays-là envoient des délégués aux conférences climatiques pour discuter de l'avenir de la planète. Il y a quelque chose d'assez surréaliste dans tout cela. Mais c'est aussi quelque chose qui est potentiellement porteur d'espoir, d'autant plus que l'impression qui se dégage de ces négociations est qu'une partie non négligeable des délégués n'y vient pas uniquement pour défendre les intérêts de leurs pays. Certains d'entre eux semblent sincèrement convaincus qu'il s'agit d'un problème urgent et important. Ils vont donc tenter de faire avancer les choses, tout en respectant les marges de manœuvre dont ils disposent. Cela vaut surtout pour les négociateurs qui participent depuis longtemps — parfois 10 , 15 , voire 20 ans - aux négociations, et qui ont avec le temps été acculturés au débat climatique. Est-ce suffisant? Certainement pas, surtout si on commence à réfléchir sérieusement aux enjeux de cette gouvernance. De toute évidence, on n'en est plus au point où l'on peut espérer résoudre la question climatique. Je pense qu'il est vraiment important de prendre la mesure de ce constat. La question climatique n'est pas un problème; il s'agit d'un ensemble d'enjeux transversaux, reliés entre eux, qui touchent tous les aspects de notre vie, et qui vont continuer à occuper nos enfants et nos petits-enfants pour les siècles à venir. Toutes les prévisions climatiques et simulations socioéconomiques le montrent. En d'autres termes, le changement climatique décrit rien de moins qu'une nouvelle condition humaine. Il n'y aura pas de grand soir du climat, mais plutôt une recherche constante de solutions partielles et de remèdes imparfaits, mais aussi des échecs et des impasses. Et même si nous réussissions - ce qu'il faut espérer - à limiter le réchauffement à un niveau à peu près tolérable, nous devrons toujours apprendre à vivre avec un climat qui change, et à gérer les effets environnementaux, économiques, sociaux et politiques de ces changements. 
La deuxième partie de votre question concerne le statut des sciences et de l'expertise. Historiquement, l'expertise a en effet d'abord eu comme fonction de sonner l'alerte. Elle a établi la réalité du changement climatique et son origine anthropique. Elle a aussi eu - et elle a encore - un rôle de mise sur agenda de nouveaux enjeux. Le «4 pour 1000 » constitue un exemple pour cela, mais aussi l'adaptation, dont l'importance a d'abord été soulignée dans les rapports du GIEC, avant que le sujet n'émerge réellement dans les négociations. L'expertise participe, ensuite, au cadrage des problèmes. Qu'on le veuille ou non, la manière dont de nouveaux enjeux sont abordés, décrits et traités dans les rapports du GIEC a des conséquences politiques non négligeables. Les gouvernements le savent très bien. Cela explique pourquoi la procédure de validation des rapports du GIEC par les délégations nationales - tout particulièrement des résumés pour décideurs, qui doivent être approuvés ligne par ligne - est souvent longue et difficile. Les savoirs scientifiques continuent donc à jouer un rôle important dans le processus politique, même s'il n'est pas toujours aisé de pointer exactement où se situe cette influence et comment elle s'opère. Une difficulté supplémentaire provient du fait que la relation entre science et politique n'est pas une relation à sens unique. Il y a des va-et-vient entre les deux sphères. Prenons l'exemple de la trajectoire de l'objectif des $1,5^{\circ} \mathrm{C}$, décrit par ma collègue Hélène Guillemot (2017) comme à la fois «nécessaire et inaccessible». À la surprise de beaucoup d'observateurs, cet objectif a dominé, pendant quelques jours, les débats à la COP21, jusqu'à être inclus dans l'accord final. Les délégués ont aussi demandé au GIEC de produire une synthèse des connaissances sur le sujet, pour mettre au clair ce que le chiffre implique en termes d'impacts et de réductions. Les scientifiques que nous avons croisés à la conférence étaient tous assez sceptiques, voire franchement consternés face à cette demande. D'un point de vue politique, l'inclusion de ce nouvel objectif leur paraissait 
paradoxale, compte tenu du fait que l'évolution des émissions globales rendait même la barre des $2^{\circ} \mathrm{C}$ de plus en plus difficile à atteindre. D'un point de vue scientifique, ils estimaient qu'une évaluation des différences entre les impacts d'un réchauffement de $2^{\circ} \mathrm{C}$ et de $1,5^{\circ} \mathrm{C}$ n'avait que peu d'intérêt. Selon eux, les prévisions existantes étaient entachées de trop d'incertitudes pour pouvoir établir des conclusions robustes sur ces différences. Depuis, le sentiment général dans les milieux scientifiques a clairement changé. Le GIEC a accepté de rédiger le rapport, qui vient d'être publiée ${ }^{20}$. Comme il est de coutume, la préparation du rapport a stimulé une intense activité scientifique, et de nouveaux travaux et projets de recherche autour de la question des $1,5^{\circ} \mathrm{C}$ ont vu le jour. Est-ce simplement du conformisme de la part des scientifiques qui ont participé à cet effort collectif? Je ne le pense pas. Si on regarde les choses autrement, on se rend compte qu'il peut en effet être intéressant, autant politiquement que scientifiquement, d'appréhender de manière plus fine ce que signifie cette différence d'un demi degré. Quelles méthodes de modélisation permettraient d'établir des estimations plus robustes sur la traduction de cette différence en termes d'impacts géographiques? Y a-t-il des processus irréversibles qui pourraient déjà être enclenchés par un dépassement des $1,5^{\circ} \mathrm{C}$ ? Quelles trajectoires d'émissions et quelles mesures politiques sont encore compatibles avec cette limite? L'objet politique des $1,5^{\circ} \mathrm{C}$ est donc désormais devenu un sujet de recherche, qui soulève de nouvelles questions et stimule de nouvelles approches. Les conclusions de ces recherches - notamment du rapport du GIEC - nourrissent en retour de nouveaux débats politiques. Il y a donc un va-et-vient continu, parfois compliqué mais souvent productif, entre travail scientifique et processus politique dans l'arène climatique.

20. Tous les rapports du GIEC sont disponibles en ligne : $<$ https://www.ipcc.ch $>$ (consulté le 02 juillet 2019). 
Question - Je reviens sur la question de la technologie, à partir de l'exemple de Kyoto. D'après mon souvenir, les Américains ne se sont pas engagés sur Kyoto parce qu'ils pensaient déjà que l'innovation technologique allait permettre de résoudre pas mal de choses. Mais de nos jours, avec la diversification des acteurs, la présence de plus en plus grande des grandes entreprises, comment va être gérée l'innovation technologique réellement liée aux émissions? Par exemple, sur les lieux d'émission très ponctuels, vont se développer des technologies lourdes pouvant avoir un impact assez fort sur l'environnement. Comment cela a-t-il été traité dans l'accord? Comment l'accord va-t-il être exploité par les gens qui veulent en faire un outil majeur de réduction, pour pratiquer les innovations technologiques pour capturer le carbone?

Stefan C. Aykut - Je dois dire que l'argument selon lequel les États-Unis auraient quitté Kyoto parce qu'ils croyaient que le problème pouvait se régler par la seule innovation technologique ne me convainc pas. Si c'était le cas, ils auraient autant pu rester dans le processus, afin de peser sur la suite des choses. C'est d'ailleurs ce qu'ils ont fait dans le cas de la lutte contre l'amincissement de la couche d'ozone. Les États-Unis étaient un acteur-clef dans la mise en place du protocole de Montréal de 1987, grâce auquel les émissions nocives pour la couche d'ozone ont pu être réduites. La position américaine sur ce dossier s'explique par le fait que les milieux économiques du pays étaient favorables à une régulation internationale. Les firmes américaines disposaient de technologies de substitution, qu'elles espéraient pouvoir exporter. Il en va de même pour la position du gouvernement allemand sur les pluies acides. Après avoir mis en place, sous la pression de son opinion publique, des régulations strictes au niveau national, le gouvernement allemand avait alors impulsé un effort réglementaire aussi au niveau européen. Cela se faisait à un moment où les industriels allemands étaient à la pointe de l'innovation dans les domaines des 
technologies «vertes», comme les filtres pour les centrales thermiques et les pots catalytiques pour les voitures. Ils espéraient donc qu'une réglementation homogène en Europe leur permettrait de conquérir de nouveaux marchés. Ces exemples montrent que l'entrée par des solutions technologiques n'est pas en soi négative. Sous certaines circonstances, elle peut contribuer à aligner les intérêts de façon à ce que l'action politique devienne possible. Ceci étant dit, il me semble que les problèmes en question - les pluies acides et l'ozone sont beaucoup moins complexes que la question climatique. Aucune solution technologique ne permettra, à elle seule, d'enrayer le réchauffement. La question est trop vaste, trop transversale, trop englobante. Elle touche à la production énergétique, à l'alimentation et aux transports, aux modes de consommation et de développement, à la régulation des marchés globaux et aux pratiques locales d'usage des terres... Des innovations technologiques seront nécessaires et bienvenues, mais il faudra aussi repenser en profondeur l'organisation de nos sociétés et de l'économie-monde. Pourra-t-on continuer, dans un monde qui risque de voir un réchauffement de 4,5 , voire $6^{\circ} \mathrm{C}$, à poursuivre un modèle économique et social fondé sur l'impératif de croissance, qui perpétue des styles de vie et des habitudes de consommation insoutenables? Poursuivre une globalisation économique qui conduit à l'augmentation continue de la circulation des biens et des personnes et présuppose la disponibilité d'une énergie abondante et bon marché? Est-il raisonnable de construire des voitures qui pèsent en moyenne 1,5 tonne, quand celles-ci ne servent, la plupart du temps, qu'à transporter une seule personne? Il y a tant de questions à se poser!

J'en viens maintenant à l'usage que les industriels pourraient faire de l'accord de Paris. Une première industrie concernée est évidemment celle des énergies renouvelables. Pour les entreprises du secteur, la COP21 aura été importante non pas tellement à cause de l'accord, qui ne contient 
rien de concret pour elles, mais parce qu'elle a redonné un certain élan aux politiques climatiques nationales. À l'instar de l'Alliance solaire internationale initiée par l'Inde, la conférence a aussi favorisé le lancement d'initiatives internationales sur les énergies renouvelables. Des conclusions analogues s'appliquent à d'autres branches qui proposent des technologies bas-carbone. À part cela, l'accord a fortement stimulé l'intérêt pour des «technologies d'émissions négatives », comme des dispositifs de captage du $\mathrm{CO}_{2}$ dans l'air (direct air capture), ou des centrales à biomasse reliées à des systèmes de capture et séquestration du carbone (bioenergy with carbon capture and storage, ou BECCS). Il y a une certaine agitation dans ces domaines, qui se manifeste dans la création de programmes de recherche et de réseaux internationaux, ainsi que dans la mise en place de projets pilotes et des premières expérimentations industrielles grandeur nature. Cette agitation provient du fait que l'article 4 de l'accord renvoie explicitement au rôle des puits de carbone pour atteindre l'objectif d'une neutralité carbone de l'économie mondiale. Pour les acteurs du domaine, ce passage constitue un levier pour justifier des projets, obtenir des financements publics ou intéresser des investisseurs privés.

Question - Une raison d'être optimiste, réside peut-être dans la société et dans les décisions individuelles que chacun doit prendre pour moins contribuer au changement climatique, et dont vous n'avez pas trop parlé. Tenez, nos accoudoirs ont tous un cendrier. Il serait inimaginable aujourd'hui de construire une salle comme celle-là avec des cendriers sur les accoudoirs. Or cette salle a environ 50 ans. Ce qui veut bien dire que les choses ont changé. Là aussi, des scientifiques ont alerté sur l'industrie du tabac, des hommes politiques ont mis en place des politiques pour dissuader de la cigarette. Des entreprises ont été terriblement impliquées, comme on le sait, dans des contre-offensives destinées à mettre en doute ce que disaient les scientifiques. Mais finalement cela a marché 
quand même. Les gens fument moins et les derniers chiffres montrent que c'est encore plus le cas des jeunes générations. De la même manière, on peut imaginer, si l'on veut être optimiste, que les jeunes générations qui sont nées avec les accords de Paris, qui ont considéré que c'étaient des enjeux majeurs, vont changer progressivement leurs modes de vie.

Stefan C. Aykut - Vous avez raison de pointer le rôle indispensable de la société civile et la nécessité d'un changement des mœurs dans les transformations à venir. Dans le débat climatique, l'importance de ces questions est souvent illustrée en mobilisant des précédents historiques, comme la lutte contre l'apartheid et l'abolition de l'esclavage. Le mouvement de désinvestissement, par exemple, s'est explicitement construit sur le modèle de la campagne contre l'apartheid en Afrique du Sud. Une alliance mondiale, composée principalement d'ONGs et d'autres acteurs de la société civile, avait alors initié, à partir des années 1960, un boycott des firmes soupçonnées de profiter de la ségrégation raciale ou de collaborer avec le gouvernement sud-africain. En isolant le régime en place sur la scène internationale et en le fragilisant économiquement, la campagne a sans doute contribué à précipiter sa chute. Elle a aussi participé d'un changement des mœurs : d'un point de vue moral, des pratiques comme la ségrégation raciale ou l'esclavage apparaissent aujourd'hui comme totalement inacceptables, au moins quand elles se présentent de manière ouverte. Mais ces exemples montrent aussi que des changements socioculturels aussi importants ne sont ni spontanés, ni automatiques. Ils ne résultent pas d'une simple prise de conscience collective d'un grand nombre d'individus. D'un point de vue sociologique, la société ne se réduit pas à l'addition des décisions individuelles. La société, c'est ce qui fait corps, qui dépasse, forme et contraint l'individu. En même temps, elle nous permet aussi d'agir collectivement, comme membres d'un groupe ou d'une organisation. La capacité d'action collective des mouvements sociaux, ONGs, 
syndicats ou partis politiques est donc indispensable pour initier, orienter et canaliser les changements sociétaux. On ne comprend ni l'abolition de l'esclavage, ni celle de l'apartheid, sans s'intéresser aux mobilisations sociales, luttes politiques et confrontations souvent violentes qui les ont accompagnées. Le constat que de tels changements sociétaux ne s'opèrent pas à partir du seul niveau individuel contraste toutefois avec la mise en avant fréquente des fameux «petits gestes du quotidien» dans le débat climatique. Le fait d'insister sur l'importance du niveau individuel est d'ailleurs typique des débats sur les problèmes écologiques. Appliqué à d'autres enjeux, ce type d'argument apparait tout de suite comme plus problématique. À titre d'exemple, il me semble que personne ici ne soutiendrait l'idée de fonder le système de sécurité sociale sur un tel principe des petits gestes, où l'aide accordée aux moins fortunés dépendrait de la bonne volonté des plus aisés. Dans ce domaine politique comme dans d'autres, on n'accepterait plus ce raisonnement-là. Je pense qu'il faut aussi le dépasser dans la lutte contre le changement climatique. Bien entendu, cela ne signifie pas que l'action individuelle ne soit pas importante ou qu'il ne faille pas faire des efforts pour réduire son empreinte écologique. Si de tels efforts enclenchent une prise de conscience sur l'insoutenabilité de nos pratiques quotidiennes et modes de consommation, très bien. Or il faut être très clair sur un point : cela ne suffira pas. On n'arrivera pas à opérer la transformation nécessaire en misant sur la seule responsabilité individuelle ou en utilisant des arguments d'ordre moral. Pour réussir cette transformation, nous avons aussi - et surtout - besoin de la capacité d'organisation et de mobilisation d'acteurs collectifs : des ONGs pour initier des campagnes et changer les termes du discours public; des mouvements sociaux pour mobiliser la société et créer des rapports de force favorables au changement; des firmes et instituts de recherche pour faciliter la transition bas-carbone par des innovations techniques; enfin, 
de l'État et de la gouvernance globale pour édicter des règles et soutenir le changement par des décisions stratégiques.

Question - Pour rebondir sur ce que vous avez dit : "Il n'y a pas que les comportements individuels qui peuvent s'additionner et faire société». Est-ce que vous pensez que la climatisation du monde pourrait toucher les accords commerciaux internationaux, et embarquer, intégrer des formes d'engagements réciproques, connecter les engagements climatiques avec d'autres aspects sur lesquels on a un peu plus de prise?

Stefan C. Aykut - Vous avez raison, on observe depuis quelque temps une volonté de coordonner davantage les activités de différentes organisations internationales. Cela passe par exemple par l'établissement de liens formels et la création de canaux de communication directe entre elles (Oberthür et Stokke, 2011). Ainsi, plusieurs organisations internationales ont un statut d'observateur dans les négociations climat - même si ce que cela implique concrètement n'est pas toujours très clair. À un niveau plus substantiel, il y a ensuite des efforts pour harmoniser différents pans du droit international et de réduire les redondances et contradictions dans la gouvernance globale. Un résultat de ces efforts est qu'un nombre croissant des traités commerciaux adoptés récemment comportent désormais des clauses environnementales. Il y a donc un certain «verdissement» du droit international du commerce, qui est à saluer (Neumayer, 2017). Pour l'instant, cette évolution reste toutefois marginale par rapport au système doctrinaire existant et ne touche pas au cœur des règles qui gouvernent la globalisation économique et financière. Elle ne suffira donc clairement pas pour contribuer de manière significative à une décarbonation de l'économie mondiale. Ceci d'autant plus que les régulations existantes entrent souvent en contradiction avec les politiques de transition. Un conflit commercial récent opposant 
l'Inde et les États-Unis illustre ces contradictions de manière paradigmatique. L'Inde, dont les émissions par tête se situent à moins d'un quart des émissions européennes, s'est engagée depuis la COP21 dans une politique de soutien à l'énergie solaire. La stratégie du gouvernement indien consiste à combiner l'objectif d'un développement bas-carbone à des objectifs sociaux et industriels, à savoir l'électrification de zones rurales non connectées au réseau et la création d'une industrie solaire nationale. Une telle stratégie est parfaitement adaptée au contexte indien, où la réduction de la pauvreté et le développement économique restent les premières priorités politiques. La création d'emplois sur le territoire national était donc vue comme une condition indispensable pour s'assurer du soutien de l'opinion publique pour une politique de transition. L'objectif ne semblait pas hors de portée : le gouvernement indien espérait que, grâce aux avantages compétitifs d'une main d'œuvre très bon marché et d'un grand marché domestique, un soutien étatique initial plutôt limité suffirait pour permettre aux entreprises indiennes du secteur de se structurer et de rattraper, à terme, leur retard sur la concurrence internationale. Une série d'appels d'offres pour la construction de centrales solaires parmi les plus puissantes au monde fut donc publiée en 2011. Ces appels contenaient une clause stipulant qu'une partie des modules et composants de centrales devait être produite en Inde. Arguant que cette clause constituait une entrave à la concurrence, les États-Unis ont attaqué l'Inde devant l'OMC deux ans plus tard. Ils ont obtenu gain de cause ${ }^{21}$ et, par la suite, l'Inde a ralenti son programme solaire. Cet épisode montre parfaitement comment le primat donné au libre-échange entrave la transformation bas-carbone des économies. La création d'industries nationales doit évidemment pouvoir

21. Les détails du jugement sont disponibles ici : < https://www.wto.org/english/ tratop_e/dispu_e/cases_e/ds456_e.htm> (consulté le 02 juillet 2019). 
faire partie d'une politique de transition. Autrement, on voit mal comment une telle politique pourrait susciter l'adhésion de l'opinion publique et le soutien des milieux économiques, qui restent indispensables pour l'inscrire dans la durée. Il est difficilement imaginable qu'un pays comme l'Inde s'engage dans une politique de transition, si celle-ci consiste à importer tous les panneaux solaires des États-Unis et de Chine.

Un deuxième exemple, pour conclure. Depuis des décennies, les secteurs du transport maritime et aérien sont parmi les sources d'émissions qui croissent le plus vite, sans qu'on puisse déceler la moindre inflexion dans cette trajectoire. Leur régulation incombe traditionnellement à deux organisations onusiennes dédiées : l'Organisation de l'aviation civile internationale et l'Organisation maritime internationale. Au moment des négociations sur la Convention climat, on a donc exclu ces domaines des discussions et délégué aux organisations compétentes la tâche de les aligner sur une trajectoire de développement bas-carbone. Depuis, rien - ou presque - ne s'est passé. Ces organisations sont notoirement réticentes à prendre des mesures qui risqueraient de contraindre l'activité économique. Et pour cause : elles sont dominées par les intérêts des secteurs qu'elles sont censées contrôler! Pour comprendre l'importance d'une régulation plus stricte du domaine, il suffit d'ailleurs de regarder les stratégies commerciales des grandes compagnies aériennes, toutes fondées sur le pronostic d'une croissance continue du nombre de passagers dans les décennies à venir. Le même pronostic soutient la construction, un peu partout dans le monde, d'aéroports nouveaux et toujours plus grands. Les villes et pays qui hébergent ces constructions mégalomanes sont engagés dans une concurrence féroce pour constituer la nouvelle plaque tournante d'un transport aérien intercontinental en pleine expansion. De toute évidence, de telles attentes et infrastructures sont totalement incompatibles avec une baisse des émissions. Si les pronostics derrière ces projets 
se matérialisent, on voit mal comment on pourra contenir le réchauffement à $2^{\circ} \mathrm{C}$. Tant que le climat ne devient pas une variable centrale dans ces domaines-là aussi, l'objectif d'une décarbonation de l'économie mondiale apparaît hors de portée. Il faudrait donc qu'il devienne inconcevable de construire de telles infrastructures sans évaluer au préalable si elles sont compatibles avec les objectifs climatiques. Sur ces questions comme sur d'autres, le processus de climatisation reste donc incomplet, partiel, sélectif. Pour pouvoir encore espérer contenir le réchauffement à un niveau à peu près tolérable, l'extension du domaine de la gouvernance climatique doit donc se poursuivre et s'intensifier, mais aussi changer de forme et de cadrage, pour dépasser le niveau discursif et s'attaquer, enfin, au cœur insoutenable de nos sociétés et de l'économie mondiale. 



\section{Références bibliographiques}

Altvater E., 2007. The social and natural environment of fossil capitalism. Socialist register, 2007, 37.

Anderson K., 2015. Duality in climate science. Nature Geoscience, 8, 898-900.

Arrouays D., Deslais W., Daroussin J., Balesdent J., Gaillard J., Dupouey J., Nys C., Badeau V., Belkacem S., 1999. Stocks de carbone dans les sols de France : quelles estimations? Comptes rendus de l'Académie d'agriculture de France, 85(6), 278-292.

Aykut S.C., 2016. Taking a wider view on climate governance: moving beyond the 'iceberg,' the 'elephant,' and the 'forest'. WIREs Climate Change. 7(3), 318-328, <http://dx.doi.org/10.1002/wcc.391> (consulté le 02 juillet 2019).

Aykut S.C., 2017. La «gouvernance incantatoire». L'accord de Paris et les nouvelles formes de gouvernance globale. lapenseeecologique. com, 1(1), <http://lapenseeecologique.com/la-gouvernance-incantatoire-laccord-de-paris-et-les-nouvelles-formes-de-gouvernance-globale/> (consulté le 02 juillet 2019).

Aykut S.C. et Castro M., 2017. The end of fossil fuels? Understanding the partial climatisation of global energy debates. In : Aykut S.C., Foyer J. et Morena E. Globalising the Climate: COP21 and the climatisation of global debates. London, Routledge Earthscan, 173-193.

Aykut S.C. et Dahan A., 2011. Le régime climatique avant et après Copenhague : sciences, politiques et l'objectif des deux degrés. Natures, Sciences, Sociétés, 19(2), 144-157.

Aykut S.C. et Dahan A., 2014. La Gouvernance du Changement Climatique : Anatomie d'un schisme de réalité. In : Pestre D. Le Gouvernement des technosciences. Gouverner le progrès et ses dégâts depuis 1945. Paris, La Découverte (Recherches), 97-132.

Aykut S.C. et Dahan A., 2015. Gouverner le climat? 20 ans de négociations internationales. Paris, Presses de Sciences Po.

Aykut S.C., Foyer J. et Morena E., 2017. Globalising the Climate: COP21 and the climatisation of global debates. London, Routledge Earthscan.

Bailey C.J., 2016. US climate change policy. London, Routledge. 
Baya-Laffite N. et Cointet J.-P., 2016. Mapping Topics in International Climate Negotiations: A Computer-Assisted Semantic Network Approach. In : Kubitschko S. et Anne K. Innovative Methods in Media and Communication Research. Cham, Switzerland, Palgrave Macmillan, 273-291.

Bodansky D., 2016. The legal character of the Paris Agreement. Review of European, Comparative \& International Environmental Law. 25(2), 142-150.

Campbell J.L., Corson C., Gray N.J., MacDonald K.I. et Brosius J.P., 2014. Studying Global Environmental Meetings to Understand Global Environmental Governance: Collaborative Event Ethnography at the Tenth Conference of the Parties to the Convention on Biological Diversity. Global Environmental Politics. 14(3), 1-20.

Dahan A., 2010. Putting the Earth System in a Numerical Box? The Evolution from Climate Modeling toward Global Change. Studies in the History and Philosophy of Modern Physics, 41, 282-292.

Dahan A. et Aykut S.C., 2012. De Rio 1992 à Rio 2012. Vingt ans de négociations climatiques : quel bilan? Quel rôle pour l'Europe? Quels futurs? Rapport pour le Centre d'analyse stratégique, Paris,

$<$ http://archives.strategie.gouv.fr/cas/system/files/121030_dahanaykut_rapport_cas_final.pdf $>$ (consulté le 02 juillet 2019).

Dahan A., Aykut S.C., Buffet C. et Viard-Crétat A., 2010. Les leçons politiques de Copenhague : Faut-il repenser le régime climatique? Koyré Climate Series, 2.

Dahan A., Aykut S.C., Guillemot H. et Korzcak A., 2009. Les arènes climatiques : forums du futur ou foires aux palabres? La Conférence de Poznan. Koyré Climate Series, 1.

Dahan A. et Guillemot, H., 2015. Les relations entre science et politique dans le régime climatique : à la recherche d'un nouveau modèle d'expertise? Natures, Sciences, Sociétés, 23, S6-S18, <http://dx.doi. org/10.1051/nss/2015014> (consulté le 02 juillet 2019).

Damian M., 2013. Mauvaise nouvelle pour le climat et les peuples de l'Amazonie équatorienne : l'abandon du projet Yasuni-ITT de gel du pétrole en terre. Natures, Sciences, Sociétés, 21(4), 428-435.

Delmas-Marty M., 2004. Le flou du droit. Paris, PUF. 
Depledge J., 2008. Striving for No: Saudi Arabia in the Climate Change Regime. Global Environmental Politics, 8(4), 9-35.

Edwards P.N., 2010. A vast Machine. Computer Models, Climate Data, and the Politics of Global Warming. Cambridge, MA, The MIT Press.

Falkner R., 2016. The Paris Agreement and the new logic of international climate politics. International Affairs, 92(5), 1107-1125.

Fournil J., Kon Kam King J., Granjou C. et Cécillon L., 2018. Le sol : enquête sur les mécanismes de (non) émergence d'un problème public environnemental. VertigO-la revue électronique en sciences de l'environnement, 18(2), <http://journals.openedition.org/vertigo/20433> (consulté le 02 juillet 2019).

Foyer J., 2015. Regards croisés sur Rio + 20 : La modernisation écologique à l'épreuve. Paris, Éditions du CNRS.

Foyer J., Aykut S.C., Morena E., 2017. Introduction: COP21 and the "climatisation" of global debates. In : Aykut S.C., Foyer J. et Morena E. Globalising the Climate: COP21 and the climatisation of global debates. London, Routledge Earthscan, 1-17.

Fuss S., Canadell J.G., Peters G.P., Tavoni M., Andrew R.M., Ciais P., Jackson R.B., Jones C.D., Kraxner F., Nakicenovic N., Le Quéré C., Raupach M.R., Sharifi A., Smith P., Yamagata Y., 2014. Betting on negative emissions. Nature Clim. Change, 4(10), 850-853, <http:// dx.doi.org/10.1038/nclimate2392> (consulté le 02 juillet 2019).

Grubb M., 2010. Copenhagen: Back to the Future. Climate Policy, $10(2), 127-130$.

Guillemot H., 2017. The necessary and inaccessible $1.5^{\circ} \mathrm{C}$ objective: a turning point in the relations between climate science and politics? In : Aykut S.C., Foyer J. et Morena E. Globalising the Climate: COP21 and the Climatisation of Global Debates. London, Routledge Earthscan, 39-56.

Hulme M. et Mahony M., 2010. Climate Change: What do we know about the IPCC? Progress in Physical Geography, 34(5), 705-718.

IEA, OPEC, OCDE et WB, 2011. Joint report by IEA, OPEC, OECD and World Bank on fossil - fuel and other energy subsidies: An update of the G20 Pittsburgh and Toronto Commitments. Paris, IEA, OCDE, OPEC, WB, <http://www.qualenergia.it/sites/default/files/articolo-doc/Fossil_subsidies_full_ODI.pdf> (consulté le 02 juillet 2019). 
Jinnah S., 2011. Climate Change Bandwagoning: The Impacts of Strategic Linkages on Regime Design, Maintenance, and Death. Global Environmental Politics, 11(3), 1-9, <https://www.mitpressjournals. org/doi/abs/10.1162/GLEP_a_00065> (consulté le 02 juillet 2019).

Joly P.-B., 2010. On the Economics of Techno-scientific Promises. In : Akrich M., Barthe Y., Muniesa F. et Mustar P. Débordements. Mélanges offerts à Michel Callon. Paris, Presse des Mines, 203-222.

Klein N., 2014. This changes everything. Capitalism vs. the climate. New York, Simon \& Schuster.

Kon Kam King J., Granjou C., Fournil J. et Cecillon L., 2018. Soil sciences and the French 4 per 1000 Initiative - The promises of underground carbon. Energy research \& social science, 45, 144-152.

Krasner S.D., 1983. International Regimes. Ithaca, NY and London, Cornell University Press.

Lahsen M., 2017. Buffers against inconvenient knowledge: Brazilian newspaper representations of the climate-meat link. $P 2 P$ e inovação, 4(1), 59-84.

Latour B., 2015. Face à Gaïa : huit conférences sur le nouveau régime climatique. Paris, La Découverte.

Little P.E., 1995. Ritual, Power and Ethnography at the Rio Earth Summit. Critique of Anthropology, 15(3), 265-288.

McGlade C. et Ekins P., 2015. The geographical distribution of fossil fuels unused when limiting global warming to $2^{\circ} \mathrm{C}$. Nature, 517(7533), 187-190, <http://dx.doi.org/10.1038/nature14016> (consulté le 02 juillet 2019).

Methmann C.P., 2010. 'Climate protection' as empty signifier: a discourse theoretical perspective on climate mainstreaming in world politics. Millennium, 39(2), 345-372.

Morgan J., Dagnet Y. et Tirpak D., 2014. Elements and ideas for the 2015 Paris agreement. ACT 2015 Working Paper. Washington, DC, World Resources Institute, <https://www.transparencypartnership.net/ sites/default/files/act_elements_ideas_fullpaper_final.pdf> (consulté le 05 octobre 2019).

Neumayer E., 2017. Greening trade and investment: environmental protection without protectionism. London, Routledge. 
Oberthür S. et Stokke O.S., 2011. Managing Institutional Complexity: Regime Interplay and Global Environmental Change. Cambridge, MA, MIT Press.

Schneider S.H. et Mesirow L.E., 1976. The Genesis Strategy. Plenum Pub Corp.

Schüssler E., Rüling C.-C. et Wittneben B.B.F., 2014. Climate Summits: The Limitations of Field-Configuring Events as Catalysts of Change in Transnational Climate Policy. Academy of Management Journal, 57(1), 140-171.

Siméant J., Sommier I. et Pommerolle M.-E., 2015. Observing Protest from a Place. The World Social Forum in Dakar (2011). Amsterdam, Amsterdam University Press.

UNEP, 2010. The Emissions Gap Report. Are the Copenhagen Accord Pledges Sufficient to Limit Global Warming to $2^{\circ} \mathrm{C}$ or $1.5^{\circ} \mathrm{C}$ ? A preliminary assessment. Nairobi, Kenya, United Nations Environment Programme.

Van Asselt H., 2016. The Role of Non-State Actors in Reviewing Ambition, Implementation, and Compliance under the Paris Agreement. Climate Law, 6(1-2), 91-108, <http://booksandjournals.brillonline.com/content/journals/10.1163/18786561-00601006> (consulté le 02 juillet 2019).

Victor D.G., 2011. Global warming gridlock: creating more effective strategies for protecting the planet. Cambridge, UK, Cambridge University Press.

Vogler J., 2016. Climate Change in World Politics. Houndmills, Basingstoke, Palgrave Macmillan.

We Mean Business, 2014. The Climate Has Changed. Why bold, low carbon action makes good business sense, <https://www.wemeanbusinesscoalition.org/blog/the-climate-has-changed/> (consulté le 02 juillet 2019).

World Bank, 2014. State and Trends of Carbon Pricing 2014. Washington, DC, World Bank.

World Bank et UNEP, 2014. Financial Institutions taking action on Climate Change, <https://www.unepfi.org/fileadmin/documents/FinancialInstitutionsTakingActionOnClimateChange.pdf $>$ (consulté le 05 octobre 2019). 



\section{Table des matières}

3 Préface

7 Introduction

11 La thèse de la climatisation : $\mathrm{du}$ constat empirique au prisme analytique

16 Les sources historiques du processus de climatisation

26 Les sommets onusiens : des machines à climatiser

32 La climatisation comme stratégie de gouvernance

37 Ambiguités et contradictions : le cas des politiques énergétiques

42 Le prix de la climatisation: les sciences des sols à l'aune du climat

48 Conclusion

51 Discussion

73 Références bibliographiques 
La permaculture ou l'art de réhabiter

L. Centimeri, 2019, 152 p.

Gouverner un monde toxique

S. Boudia, N. Jas, 2019, 124p.

Penser l'éventuel

Faire entrer les craintes dans le travail scientifique

N. Bouleau, 2017, 214 p.

Science et territoires de l'ignorance

M. Girel, 2017, 156 p.

L'emprise des droits intellectuels sur le monde vivant

M.-A. Hermitte, 2016, 150 p.

La science au pluriel

Essai d'épistémologie pour des sciences impliquées

L. Coutellec, 2015, 88 p.

Le marché aux connaissances

L. Busch, 2014, 160 p.

Nature à vendre

V. Maris, 2014, 94 p.

Pour une interprétation littéraire des controverses scientifiques

Y. Citton, 2013, 180 p.

Désacraliser le chiffre dans l'évaluation du secteur public

A. Ogien, 2013, $119 \mathrm{p}$.

La recherche malade du management

V. de Gaulejac, 2012, 96 p.

L'ontophylogenèse

J.-J. Kupiec, 2012, 80 p.

Les sciences face aux créationnismes

G. Lecointre, 2012, 176 p.

Éthique et recherche

J.-F. Théry, J.-M. Besnier, E. Hirsch, 2011, 64 p. 
L'écologie des autres

L'anthropologie et la question de la nature

P. Descola, 2011, 112 p.

\section{Expérimentation animale}

J.-P. Marguénaud, 2011, 78 p.

Biotechnologie, nanotechnologie, écologie

Entre science et idéologie

M.-H. Parizeau, 2010, 88 p.

De la protection de la nature au pilotage de la biodiversité

P. Blandin, 2009, 124 p.

Complexité aléatoire et complexité organisée J.-P. Delahaye, 2009, 76 p.

Science et communication : pour le meilleur ou pour le pire?

M. Claessens, 2009, 176 p.

Penser comme un rat

V. Despret, 2009, 96 p.

À quoi sert l'histoire des sciences?

M. Morange, 2008, 72 p.

\section{Agronomes et paysans}

Un dialogue fructueux

A. Pochon, 2008, 72 p.

L'analyse des risques

L'expert, le décideur et le citoyen

B. Chevassus-au-Louis, 2007, 96 p

Se libérer de la matière ?

Fantasmes autour des nouvelles technologies

B. Bensaude-Vincent, 2004, 94 p.

L'évaluation du travail à l'épreuve du réel

Critique des fondements de l'évaluation

C. Dejours, 2003, 84 p.

Science, argent et politique

Un essai d'interprétation

D. Pestre, 2003, 204 p. 
Mise en page : Hélène Bonnet - Studio 9

Impression : IsiPrint

Dépôt légal : janvier 2020 


\section{Science $\int_{n \text { questions }}^{S}$}

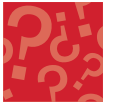

La question climatique s'est diffusée dans de nombreuses sphères de la vie publique, forçant des acteurs parfois assez éloignés des enjeux écologiques à s'y intéresser. Un nombre croissant de firmes, d'associations et d'institutions se voient désormais contraints à repenser leurs orientations stratégiques, leurs routines organisationnelles et leurs pratiques économiques.

L'auteur propose de saisir les évolutions en cours comme le résultat d'une " climatisation » du monde. Cette expression traduit la capacité du changement climatique à connecter et à agréger toutes sortes de sujets aussi divers que la sécurité alimentaire, la finance ou les sols.

Paradoxalement, cette force d'attraction rend la formation des politiques climatiques de plus en plus complexe. En décryptant la gouvernance climatique instaurée notamment dans les Conferences of Parties, les COP, Stefan C. Aykut aide également à en cerner les effets ambigus et contradictoires.

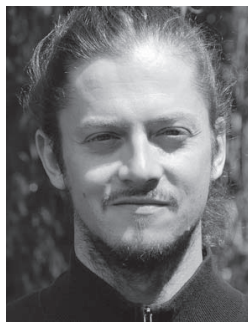

Stefan Ciran Aykut est sociologue et politiste, professeur junior à l'université de Hambourg. Il a étudié à Berlin (Freie Universität), à Istanbul (Sabanci University) et à Paris (EHESS). Ses recherches visent à comprendre les transformations contemporaines des modes de gouvernement et d'organisation sociale face à la crise écologique globale. Il travaille plus particulièrement sur la gouvernance globale du climat, les transitions énergétiques en France et en Allemagne et l'institutionnalisation de limites écologiques globales dans différentes sphères sociales.

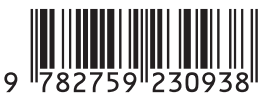

